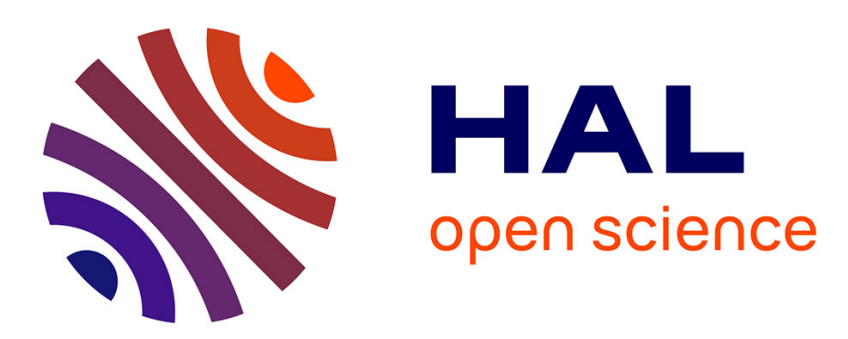

\title{
Effet photoréfractif dans les cristaux d'oxyde de bismuth et de silicium (Bi12SiO20) ou de germanium (Bi12GeO20)
}

Gérald Roosen, Gilles Le Saux, Gilles Pauliat, Michel Allain, Jean-Michel Jonathan, Alain Brun

\section{To cite this version:}

Gérald Roosen, Gilles Le Saux, Gilles Pauliat, Michel Allain, Jean-Michel Jonathan, et al.. Effet photoréfractif dans les cristaux d'oxyde de bismuth et de silicium (Bi12SiO20) ou de germanium (Bi12GeO20). Revue de Physique Appliquée, 1987, 22 (10), pp.1253-1267. 10.1051/rphysap:0198700220100125300 . jpa-00245675

\section{HAL Id: jpa-00245675 https://hal.science/jpa-00245675}

Submitted on 1 Jan 1987

HAL is a multi-disciplinary open access archive for the deposit and dissemination of scientific research documents, whether they are published or not. The documents may come from teaching and research institutions in France or abroad, or from public or private research centers.
L'archive ouverte pluridisciplinaire HAL, est destinée au dépôt et à la diffusion de documents scientifiques de niveau recherche, publiés ou non, émanant des établissements d'enseignement et de recherche français ou étrangers, des laboratoires publics ou privés. 


\title{
Effet photoréfractif dans les cristaux d'oxyde de bismuth et de silicium $\left(\mathrm{Bi}_{12} \mathrm{SiO}_{20}\right)$ ou de germanium $\left(\mathrm{Bi}_{12} \mathrm{GeO}_{20}\right)$
}

\author{
G. Roosen, G. Le Saux, G. Pauliat, M. Allain, J. M. C. Jonathan et A. Brun
}

(Reçu le 24 décembre 1986, accepté le 4 mars 1987)

\begin{abstract}
Résumé. - L'effet photoréfractif permet d'induire optiquement dans les matériaux électro-optiques et photoconducteurs, des variations d'indice en utilisant des faisceaux lumineux de faible puissance. Mis en évidence il y a une vingtaine d'années, ce phénomène a connu un regain d'intérêt grâce à la conjugaison de phase et à ses applications potentielles en traitement optique des images. Dans cet article, et en nous limitant aux cristaux sillénites nous décrirons le mécanisme de cet effet et en préciserons les caractéristiques en régime d'excitation quasi continue puis nanoseconde. Nous évoquerons en conclusion certaines applications liées aux propriétés spécifiques de l'effet photoréfractif.
\end{abstract}

\begin{abstract}
The photorefractive effect allows the optical generation in electro-optic and photoconductive materials of index variations, with very low energy levels. Described twenty years ago, the effect recently gain interest due to its use for optical processing and optical phase conjugation. In the case of sillenite crystals, we presently summarize the physics involved in the photorefractive effect under both quasi continuous and short pulse illuminations. Caracterizations of the samples are derived and specific applications are proposed.
\end{abstract}

\section{Introduction.}

Un effet photoréfractif important est souvent observé dans les cristaux ferroélectriques $\left(\mathrm{LiN}_{\mathrm{b}} \mathrm{O}_{3}, \mathrm{BaTiO}_{3}, \ldots\right)$, les sillénites $\left(\mathrm{Bi}_{12} \mathrm{SiO}_{20}\right)$ ou les «semiconducteurs » (GaAs dopé $\mathrm{Cr}$, InP dopé Fe...) [1-3]. Ces matériaux, isolants ou semi-isolants dans le noir, sont à la fois photoconducteurs et électro-optiques.

Considérons un tel cristal, illuminé par la distribution d'intensité résultant de l'interférence de deux ondes planes. Des électrons (ou des trous) sont excités dans la bande de conduction (ou de valence) à partir de niveaux donneurs situés dans la bande interdite (Fig. 1). Il en résulte une modulation spatiale simultanée des densités de porteurs libres et d'ions dans le réseau (Fig. 2a). Par diffusion ou entraînement sous l'effet d'un champ électrique, les porteurs libres migrent jusqu'à leur recombinaison. Il se crée alors un réseau de champ de charge d'espace lié au réseau d'ions et déphasé d'un angle $\psi$ par rapport à la figure d'interférence (Fig. 2b). Le champ électrique qui en résulte engendre, par effet électro-optique, un réseau d'indice de réfraction (Fig. 2c).

Bien que sommaire, cette description permet de

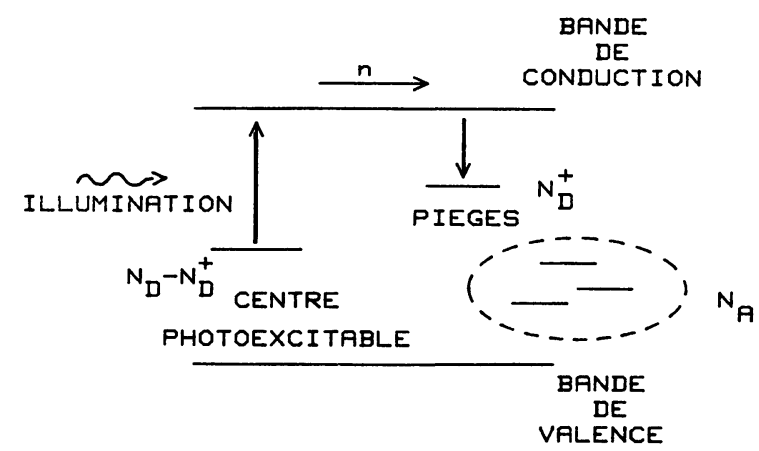

Fig. 1. - Photoexcitation, migration et recombinaison des porteurs de charge (électrons).

[Photoexcitation, transport and recombination of charge carriers (electrons).]

dégager les caractéristiques essentielles du phénomène :

- l'effet photoréfractif est sensible à l'énergie reçue et non à l'intensité. Cela permet d'envisager l'utilisation de lasers continus et de faible puissance dans des expériences d'optique non linéaire,

- il s'agit d'un effet non local. Le déphasage du réseau d'indice par rapport à la figure d'interférence 


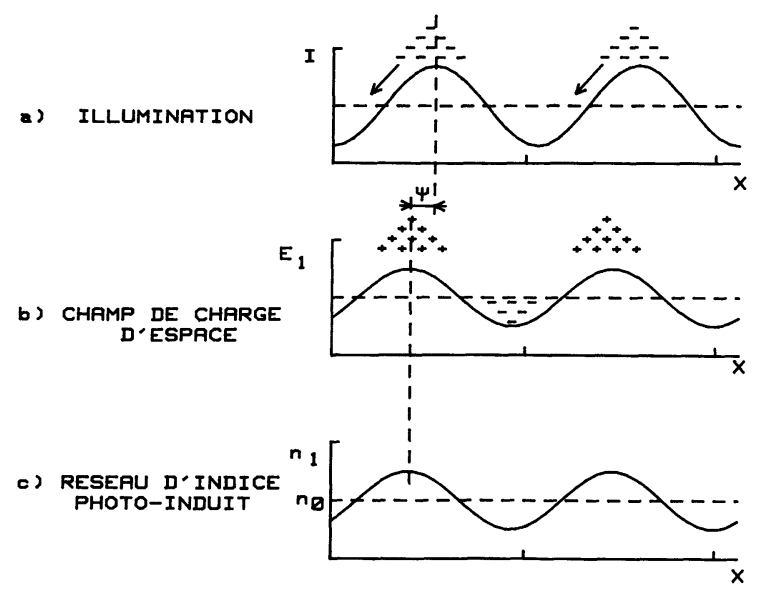

Fig. 2. - Création d'un réseau d'indice à partir d'une figure d'interférence dans un cristal photoréfractif : cas de la conduction par électrons.

[Writing of an index grating from an interference pattern in a photorefractive crystal (electron conductivity).]

permet en particulier le transfert d'énergie par couplage entre deux ondes de même fréquence,

- le phénomène est totalement réversible; tout réseau peut être effacé par une illumination uniforme du cristal,

- ces matériaux possèdent un effet mémoire: dans l'obscurité, la distribution de charge est figée et le réseau persiste. Cette durée de mémorisation peut atteindre plusieurs mois et dépend de la conductivité du cristal dans le noir.

Ces quatre propriétés simultanées expliquent l'intérêt suscité par les cristaux photoréfractifs tant dans le traitement optique des images qu'en conjugaison de phase [4]. Nous citerons en particulier :

- l'interférométrie holographique en temps réel [5] ;

- le stockage optique de l'information [6] ;

- les fonctions logiques [7-9] ;

- les cavités laser auto-oscillantes [10-12] ;

- les mémoires associatives [13-15] ;

- les interconnexions dynamiques [16, 17].

Les cristaux de la famille des sillénites ont l'avantage d'être disponibles en échantillons de grandes dimensions (plusieurs $\mathrm{cm}^{3}$ ) avec une bonne qualité optique. Leur sensibilité est élevée : une énergie d'une centaine de micro-joules par centimètre carré suffit, en régime continu, à inscrire un réseau d'efficacité égale à $1 \%$; en régime d'impulsions nanosecondes, la même efficacité est obtenue pour une énergie de $1 \mathrm{~mJ} / \mathrm{cm}^{2}$.

Après une présentation rapide des propriétés physiques de ces cristaux, nous décrirons l'établissement du réseau photoréfractif soit par des excitations lumineuses de longue durée et de faible puissance soit par des excitations plus brèves (impulsions nanosecondes). Nous discuterons l'influence des conditions expérimentales et des paramètres introduits dans le modèle et présenterons des méthodes optiques permettant leur détermination.

En conclusion, nous indiquerons quelques applications éventuelles de ces cristaux pour la logique optique, les mémoires associatives et l'interconnexion dynamique.

\section{Propriétés physiques.}

Les oxydes de bismuth et de germanium (BGO) et ceux de bismuth et de silicium (BSO), cristallisent dans une structure cubique centrée. La maille élémentaire du BGO comporte par exemple deux motifs $\mathrm{Bi}_{12} \mathrm{GeO}_{20}$. Son paramètre de maille vaut $10,145 \AA(10,104 \AA$ pour le BSO). L'étude aux rayons $\mathrm{X}$ et l'existence de l'activité optique montrent l'appartenance de ces cristaux à la classe de symétrie 23 [18].

La technique de croissance utilisée est celle de Czochralski $[19,20]$. On mélange intimement et en proportions stœchiométriques, les oxydes $\mathrm{Bi}_{2} \mathrm{O}_{3}$ et $\mathrm{GeO}_{2}$ (auxquels on ajoute éventuellement un dopant tel que $\mathrm{Fe}_{2} \mathrm{O}_{3}$ ). Le mélange obtenu est ensuite porté à la température de fusion $\left(930^{\circ} \mathrm{C}\right)$ dans un creuset en platine. A partir de cet état fondu, on produit un amorçage de la croissance au moyen d'un germe monocristallin orienté et mis en contact avec le liquide. On tire ensuite le porte-germe lentement suivant un axe vertical. Après croissance, les cristaux sont recuits ce qui a pour effet de relâcher les contraintes.

Les cristaux photoréfractifs de BSO et BGO ont une énergie de bande interdite voisine de $3,2 \mathrm{eV}$ [21]. Différentes techniques d'études (spectre d'absorption, de photoconductivité $[21,22]$; méthode des courants stimulés thermiquement [23] ; spectroscopie par transitoires de courants photoinduits [24] ; spectre de photoluminescence [25, 26]) ont permis de tracer, pour des cristaux non dopés, le diagramme des transitions optiques de la figure 3 [22]. La densité en centres donneurs $N_{\mathrm{D}}$ $\left(N_{\mathrm{D}} \cong 10^{19} \mathrm{~cm}^{-3}\right)$ incite certains auteurs à identifier ces centres à des défauts cristallins [21]. Certains atomes de bismuth ionisés $\mathrm{Bi}^{3+}$ associés à un défaut d'électrons occuperaient des sites d'oxygène tétraédrique à la place du germanium $\mathrm{Ge}^{4+}$.

Les courbes de photoconduction du BSO en fonction de la longueur d'onde (Fig. 4) montrent deux «pics»[22]. Le premier est attribué à une transition directe de la bande de valence à la bande de conduction $(3,2 \mathrm{eV}$ à température ambiante) et le second, voisin de $2,6 \mathrm{eV}$, à une transition indirecte à partir de centres donneurs situés à $2,2 \mathrm{eV}$ sous la bande de conduction. Une autre caractéristique importante de ces cristaux est leur très grande 


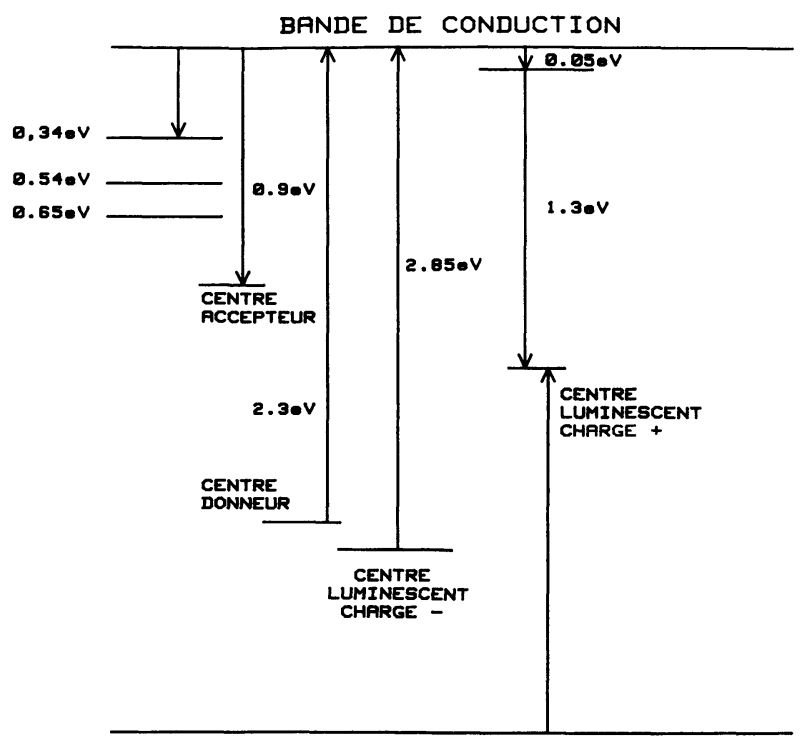

Bande de Valence

Fig. 3. - Diagramme des transitions optiques d'un cristal de BSO.

[Optical transitions in BSO crystals.]

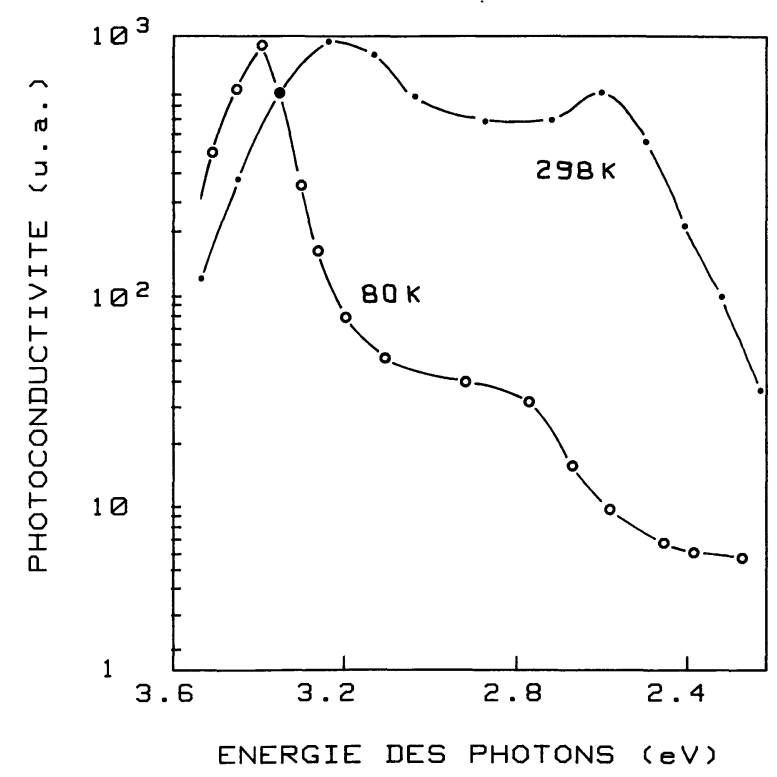

Fig. 4. - Photoconduction du BSO en fonction de l'énergie des photons.

[Photoconductivity in BSO as a function of photon energy.]

conductivité sous illumination par rapport à celle observée dans le noir. Par exemple [6] :

- pour un BSO :

$$
\begin{aligned}
\sigma=\left[\Omega^{-1} \mathrm{~cm}^{-1}\right]=1,6 \times 10^{-15}+ \\
+7,3 \times 10^{-8} I_{0}\left[\mathrm{~W} \mathrm{~cm}^{-2}\right]
\end{aligned}
$$

- pour un BGO :

$\sigma=\left[\Omega^{-1} \mathrm{~cm}^{-1}\right]=3 \times 10^{-14}+$

$$
+5,7 \times 10^{-8} I_{0}\left[\mathrm{~W} \mathrm{~cm}^{-2}\right]
$$

avec $I_{0}$ l'intensité incidente pour une longueur d'onde de $514 \mathrm{~nm}$.

\section{Etablissement du champ de charge d'espace.}

Deux modèles distincts ont été développés pour rendre compte de l'évolution temporelle d'un réseau photoinduit dans un cristal photoréfractif. Ils diffèrent par le mécanisme responsable du transport de charge. Pour l'un [27] (« Hopping model»), les porteurs de charge sautent directement d'un site à un autre avec une probabilité de saut dépendant de la différence de potentiel entre ces deux sites. Pour l'autre modèle [28], le transfert de charge se fait par l'intermédiaire des porteurs libres (entraînement sous champ et diffusion). Des études quantitatives [29] ont montré que, dans le cas des cristaux de BSO, seul ce second modèle était compatible avec les faits expérimentaux. De plus, le «Hopping model " qui ne prend pas en compte les porteurs libres ne peut décrire tous les phénomènes observés en régime impulsionnel $[30,31]$.

Dans un but de clarté, nous développerons le modèle de transport de charge en faisant les hypothèses suivantes (Fig. 1) :

- seuls les électrons, de densité $n$ par unité de volume, participent au transport de charge ;

- ces électrons sont produits par excitation lumineuse à partir d'un seul type de centres donneurs de densité $N_{\mathrm{D}}$.

Si $N_{\mathrm{D}}^{+}$est la densité de donneurs déjà ionisés, le taux de production est $s I\left(N_{\mathrm{D}}-N_{\mathrm{D}}^{+}\right)$(avec $I$ l'intensité lumineuse, $s$ la section efficace de photoionisation). Les électrons de la bande de conduction se recombinent dans les centres $N_{\mathrm{D}}^{+}$avec une constante de temps $\tau_{\mathrm{R}}$ liée à $N_{\mathrm{D}}^{+}$par :

$$
\tau_{\mathrm{R}}=\frac{1}{\gamma N_{\mathrm{D}}^{+}} .
$$

L'existence des centres $N_{\mathrm{D}}^{+}$en l'absence d'illumination est due à la présence de pièges profonds. Ces sites de densité $N_{\mathrm{A}}$ (Fig. 1) ont une charge négative qui assure la neutralité électrique du cristal. En l'absence d'illumination, on a : $N_{\mathrm{D}}^{+}=N_{\mathrm{A}}$. On admet que ces centres $N_{\mathrm{A}}$ ne sont pas photoexcitables. Peu importe donc leur niveau d'énergie et le nombre de types distincts de centres $N_{\mathrm{A}}$. Seul intervient leur densité totale.

On écrit alors :

- l'équation de génération des donneurs ionisés :

$$
\frac{\partial N_{\mathrm{D}}^{+}}{\partial t}=s I\left(N_{\mathrm{D}}-N_{\mathrm{D}}^{+}\right)-\gamma n N_{\mathrm{D}}^{+},
$$


- l'équation de transport de charge régie par la loi d'Ohm et la diffusion :

$$
\mathbf{j}=e n \mu \mathbf{E}+\mu k_{\mathrm{B}} T \operatorname{grad} n,
$$

(avec $\mathbf{j}$ la densité de courant, $e$ la valeur absolue de la charge de l'électron, $\mu$ celle de sa mobilité, $k_{\mathrm{B}}$ la constante de Boltzmann et $T$ la température absolue),

- l'équation de continuité :

$$
\frac{\partial n}{\partial t}=\frac{\operatorname{div} \mathbf{j}}{e}+\frac{\partial N_{\mathrm{D}}^{+}}{\partial t},
$$

- l'équation de Poisson:

$$
\operatorname{div}\left(\varepsilon_{0} \varepsilon_{\mathrm{s}} \mathrm{E}\right)=-e\left(n+N_{\mathrm{A}}-N_{\mathrm{D}}^{+}\right)
$$

avec $\varepsilon_{0} \varepsilon_{s}$, la permittivité statique.

Illuminons le cristal par une distribution d'intensité $I(x)$, sinusoïdale selon $x$, de modulation $m$ et de vecteur d'onde $\mathbf{k}_{R}$ :

$$
I(x)=I\left[1+m \mathcal{R}_{\mathrm{e}}\left\{\exp \left[i k_{\mathrm{R}} x\right]\right\}\right] .
$$

Le système d'équations (1) à (4) est linéarisable lorsque la modulation $m$ est petite devant l'unité (cela permet de ne tenir compte que des termes d'ordres zéro et un en fréquence spatiale). Les grandeurs à considérer s'écrivent alors :

$$
\left\{\begin{aligned}
N_{\mathrm{D}}^{+}(x, t) & =N_{\mathrm{D} 0}^{+}(t)+\mathcal{R}_{\mathrm{e}}\left[N_{\mathrm{D} 1}^{+}(t) \exp \left(i k_{\mathrm{R}} x\right)\right] \\
n(x, t) & =n_{0}(t)+\mathcal{R}_{\mathrm{e}}\left[n_{1}(t) \exp \left(i k_{\mathrm{R}} x\right)\right] \\
j(x, t) & =j_{0}(t)+\mathcal{R}_{\mathrm{e}}\left[j_{1}(t) \exp \left(i k_{\mathrm{R}} x\right)\right] \\
E(x, t) & =E_{0}(t)+\mathcal{R}_{\mathrm{e}}\left[E_{1}(t) \exp \left(i k_{\mathrm{R}} x\right)\right]
\end{aligned}\right.
$$

Dans les cristaux non centro-symétriques tels que le $\mathrm{BSO}$, le champ de charge d'espace $E_{1}$ engendre par effet électro-optique linéaire (effet Pockels) une modulation périodique de la permittivité du matériau, c'est-à-dire, un réseau d'indice. Par définition de l'effet Pockels, on a :

$$
\left[\varepsilon_{1}\right]=-[\varepsilon]\left[[\mathbf{R}] \cdot \mathbf{k}_{\mathrm{R}}\right][\varepsilon] E_{1}
$$

où $[\varepsilon]$ est le tenseur de permittivité relative aux fréquences optiques et $[R]$ est le tenseur électrooptique. Pour les matériaux de la classe de symétrie 23 (ou $43 \mathrm{~m}$ ), ce tenseur de rang 3 s'écrit dans le repère des axes cristallographiques et en utilisant les notations contractées habituelles [32] :

$$
[\mathbf{R}]=\left[\begin{array}{ccc}
0 & 0 & 0 \\
0 & 0 & 0 \\
0 & 0 & 0 \\
r_{41} & 0 & 0 \\
0 & r_{52} & 0 \\
0 & 0 & r_{63}
\end{array}\right] \text { avec } r_{41}=r_{52}=r_{63}
$$

L'analyse de l'onde résultant de la diffraction d'un faisceau lumineux par cette modulation d'indice, permet l'étude du champ de charge d'espace $E_{1}$.

On remarque que l'équation de Poisson (Eq. 4), introduit un déphasage de $\pm \pi / 2$ (selon le signe des porteurs) entre le champ de charge d'espace et la densité de charge. L'application d'un champ électrique externe introduit un déphasage entre cette densité de charge et la distribution d'intensité lumineuse (Eq. (5)). Si $\psi$ désigne alors le déphasage total entre le champ de charge d'espace et la figure d'interférence (Fig. 2), le déphasage $\psi^{\prime}$ du réseau d'indice sera égal à $\psi$ ou $\psi+\pi$ selon le signe du produit $[R] \times \mathbf{k}_{R}$ dans l'équation (7). Ce déphasage permet un transfert d'énergie (couplage) entre deux ondes, par diffraction sur le réseau de phase qu'elles créent (Fig. 5).

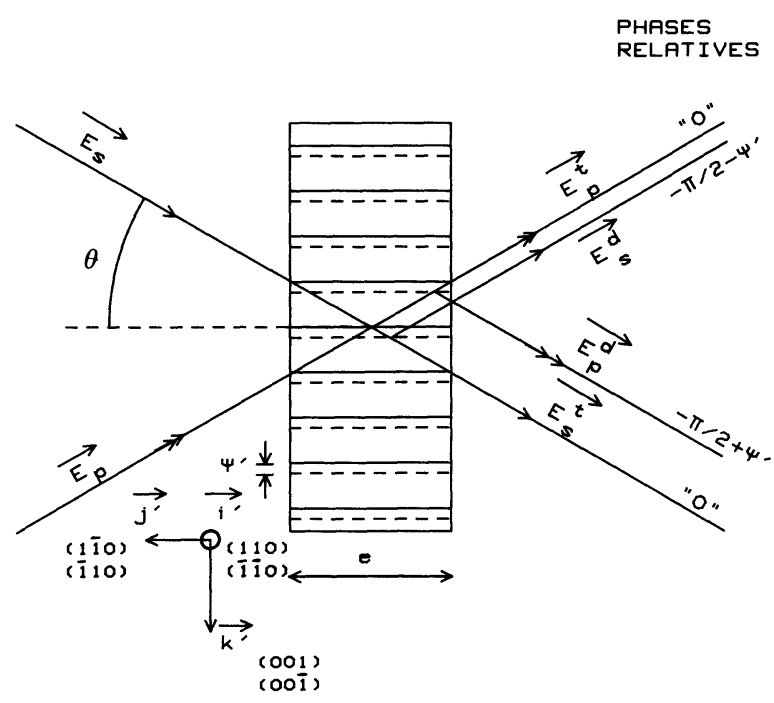

Fig. 5. - Schéma de principe du couplage entre deux ondes pompe $\left(E_{\mathrm{p}}\right)$ et sonde $\left(E_{\mathrm{s}}\right)$ dans un cristal photorefractif.

[Beam coupling in a photorefractive crystal between a signal beam $\left(E_{\mathrm{s}}\right)$ and a pump beam $\left(E_{\mathrm{p}}\right)$.]

Chacune des ondes transmises par le cristal résulte de la superposition d'une onde transmise $E^{\mathrm{t}}$ et d'une onde difffractée $E^{\mathrm{d}}$. L'onde diffractée par un réseau de phase est toujours en avance de $\pi / 2$ par rapport à l'onde transmise. A ce déphasage s'ajoute le déphasage $\psi^{\prime}$ d'où une différence de phase totale $-\pi / 2-\psi^{\prime}$ entre $E_{\mathrm{p}}^{\mathrm{t}}$ et $E_{\mathrm{s}}^{\mathrm{d}}$ et $-\frac{\pi}{2}+\psi^{\prime}$ entre $E_{\mathrm{s}}^{\mathrm{t}}$ et $E_{\mathrm{p}}^{\mathrm{d}}$ (Fig. 5). Ces ondes sont cohérentes et interfèrent. S'il n'y a pas de champ électrique appliqué au cristal, le déphasage $\psi^{\prime}$ vaut $\pm \pi / 2$ selon le signe des porteurs de charge et celui du coefficient électro-optique. Les interférences sont donc constructives sur une voie et destructives sur l'autre. C'est là un point essentiel pour la détermination du signe des porteurs de charge responsables de l'effet photoréfractif.

La résolution analytique du système d'équa- 
tions (6), difficile dans le cas général, se simplifie dans certains cas particuliers :

i) lorsque la durée de l'illumination est très longue devant tous les temps caractéristiques (diffusion, entraînement, recombinaison) ;

ii) lorsque la durée de l'illumination est très courte devant le temps de recombinaison des porteurs libres.

\section{Effet photoréfractif en régime d'excitation quasi continue.}

L'illumination est supposée suffisamment faible pour que le temps d'établissement de l'effet photoréfractif soit grand devant le temps de recombinaison des porteurs de charge. Par suite, les grandeurs non modulées spatialement $\left(N_{\mathrm{D} 0}^{+}, n_{0}, j_{0}\right)$ sont indépendantes du temps et $n_{0} \ll N_{\mathrm{A}}$. Le champ électrique $E_{0}$ appliqué est lui-même statique.

Dans ces conditions, la résolution des équations (1) à (6) conduit à l'équation d'évolution du champ de charge d'espace $E_{1}[33]$ :

$$
\begin{aligned}
\frac{\partial^{2} E_{1}}{\partial t^{2}}+ & \frac{\partial E_{1}}{\partial t}\left[\frac{-i}{\tau_{\mathrm{E}}}+\frac{1}{\tau_{\mathrm{DI}}}+\frac{1}{\tau_{1}}+\frac{1}{\tau_{\mathrm{R}}}+\frac{1}{\tau_{\mathrm{D}}}\right]+ \\
+ & {\left[E_{1}-m E_{\mathrm{sc}} e^{-i \psi}\right] \times } \\
& \times\left[\frac{-i}{\tau_{\mathrm{E}} \tau_{\mathrm{I}}}+\frac{1}{\tau_{\mathrm{D}} \tau_{\mathrm{I}}}+\frac{1}{\tau_{\mathrm{R}} \tau_{\mathrm{DI}}}\right]=0
\end{aligned}
$$

avec :

$\tau_{\mathrm{E}}=\frac{1}{k_{\mathrm{R}} \mu E_{0}}$,

$\tau_{\mathrm{DI}}=\frac{\varepsilon_{0} \varepsilon_{\mathrm{s}}}{e \mu n_{0}}$ :

constante de temps de relaxation diélectrique .

$\tau_{\mathrm{I}}=\frac{1}{s I+\gamma n_{0}}$

$\tau_{\mathrm{R}}=\frac{1}{\gamma N_{\mathrm{A}}}:$

constante de temps de recombinaison.

$\tau_{\mathrm{D}}=\frac{e}{k_{\mathrm{B}} \mu T k_{\mathrm{R}}^{2}}:$

constante de temps de diffusion .

A l'état stationnaire, le champ de charge d'espace s'écrit :

$$
E_{1}=+m E_{\mathrm{sc}} e^{i \psi}
$$

Le déphasage $\psi$ du champ de charge d'espace par rapport à la figure d'illumination et son module $E_{\mathrm{sc}}$ s'expriment en fonction des différentes constan- tes de temps ou en fonction des champs électriques caractéristiques qui sont :

- $E_{0}$ le champ appliqué au cristal,

- $E_{\mathrm{D}}$ le champ de diffusion : $E_{\mathrm{D}}=\frac{k_{\mathrm{B}} T k_{\mathrm{R}}}{e}$,

- $E_{\mathrm{q}}$ qui représente le champ maximal que l'on peut photo-induire dans le cristal :

$$
\begin{gathered}
E_{\mathrm{q}}=\frac{e N_{\mathrm{A}}}{\varepsilon_{0} \varepsilon_{s} k_{\mathrm{R}}} \cdot \frac{N_{\mathrm{D}}-N_{\mathrm{A}}}{N_{\mathrm{D}}} . \\
E_{\mathrm{sc}}=E_{\mathrm{q}}\left[\frac{E_{0}^{2}+E_{\mathrm{D}}^{2}}{E_{0}^{2}+\left(E_{\mathrm{D}}+E_{\mathrm{q}}\right)^{2}}\right]^{1 / 2} \\
\cos \psi=-\frac{E_{0} E_{\mathrm{q}}}{\left[\left(E_{\mathrm{D}}+E_{q}\right)^{2}+E_{0}^{2}\right]^{1 / 2}\left[E_{0}^{2}+E_{\mathrm{D}}^{2}\right]^{1 / 2}} \\
\sin \psi=-\frac{E_{\mathrm{D}}\left(E_{\mathrm{D}}+E_{\mathrm{q}}\right)+E_{0}^{2}}{\left[\left(E_{\mathrm{D}}+E_{\mathrm{q}}\right)^{2}+E_{0}^{2}\right]^{1 / 2}\left[E_{0}^{2}+E_{\mathrm{D}}^{2}\right]^{1 / 2}} .
\end{gathered}
$$

L'équation d'évolution du champ de charge d'espace (9) est une équation différentielle du second ordre. Il existe donc deux constantes de temps d'évolution du champ, $\tau_{+}$et $\tau_{-}$, en général complexes. Cependant la partie réelle de la constante de temps $\tau_{+}$est indépendante du champ électrique appliqué et reste toujours inférieure ou égale à $\tau_{R}$ [34]. On ne prend donc en considération que la constante de temps longue $\tau_{-}$et l'équation (9) s'écrit :

$$
\frac{\partial E_{1}}{\partial t}+\frac{1}{\tau_{-}}\left[E_{1}-m E_{\mathrm{sc}} e^{i \psi}\right]=0
$$

C'est l'expression habituellement obtenue en négligeant dès le départ l'évolution temporelle de la population électronique modulée et sa contribution au champ de charge d'espace. Les ordres de grandeur des différentes constantes de temps mesurées sur les échantillons montrent que lors d'une excitation lumineuse avec un laser continu, on a toujours $\frac{1}{\tau_{\mathrm{I}}} \ll \frac{1}{\tau_{\mathrm{D}}}$. Par contre, l'approximation $\frac{1}{\tau_{\mathrm{DI}}} \ll \frac{1}{\tau_{\mathrm{D}}}$ n'est vérifiée que pour des illuminations faibles. Alors $\tau_{-}$s'exprime sous la forme $\frac{1}{\tau_{-}}=\frac{1}{\tau}+i \omega$, avec :

$$
\begin{gathered}
\omega=\frac{1}{\tau_{\mathrm{DI}}} \cdot \frac{\kappa^{2}}{k_{\mathrm{D}}^{2}} \cdot\left(\frac{\left(\kappa^{2}-k_{\mathrm{D}}^{2}\right)\left(\frac{k_{\mathrm{R}} E_{0} e}{k_{\mathrm{B}} T}\right)}{\left(k_{\mathrm{R}}^{2}+\kappa^{2}\right)^{2}+\left(\frac{k_{\mathrm{R}} E_{0}}{k_{\mathrm{B}} T}\right)^{2}}\right) \\
\tau=\tau_{\mathrm{DI}} \cdot \frac{\kappa_{\mathrm{D}}^{2}}{\kappa^{2}} \cdot \frac{\left(k_{\mathrm{R}}^{2}+\kappa^{2}\right)^{2}+\left(\frac{k_{\mathrm{R}} E_{0} e}{k_{\mathrm{B}} T}\right)^{2}}{\left(k_{\mathrm{R}}^{2}+k_{\mathrm{D}}^{2}\right)\left(k_{\mathrm{R}}^{2}+\kappa^{2}\right)+\left(\frac{k_{\mathrm{R}} E_{0} e}{k_{\mathrm{B}} T}\right)^{2}}
\end{gathered}
$$


où :

$-\kappa=\frac{1}{L}=k_{\mathrm{R}} \sqrt{\frac{\tau_{\mathrm{D}}}{\tau_{\mathrm{R}}}}=\sqrt{\frac{e}{\mu k_{\mathrm{B}} T \tau_{\mathrm{R}}}}$ l'inverse de la longueur de diffusion $L$ des électrons dans la bande de conduction,

$-k_{\mathrm{D}}=\frac{1}{L_{\mathrm{D}}}=k_{\mathrm{R}} \sqrt{\frac{\tau_{\mathrm{I}} \tau_{\mathrm{D}}}{\tau_{\mathrm{R}} \tau_{\mathrm{DI}}}}=\sqrt{\frac{N_{\mathrm{A}} e^{2}}{\varepsilon_{0} \varepsilon_{s} k_{\mathrm{B}} T}} \quad$ l'in verse de la longueur d'écran de Debye.

Ces expressions séparent les paramètres expérimentaux des paramètres du matériau $\left(\kappa_{\mathrm{I}}, k_{\mathrm{D}}\right.$ et $\left.\tau_{\mathrm{DI}} \cdot I\right)$ qui interviennent dans les conditions requises par le modèle $[29,35,36]$.

4.1 INFLUENCE D'UN CHAMP ÉLECTRIQUE EXTERNE SUR LE CHAMP DE CHARGE D'ESPACE. - La figure 6 qui décrit l'évolution de l'efficacité de diffraction en

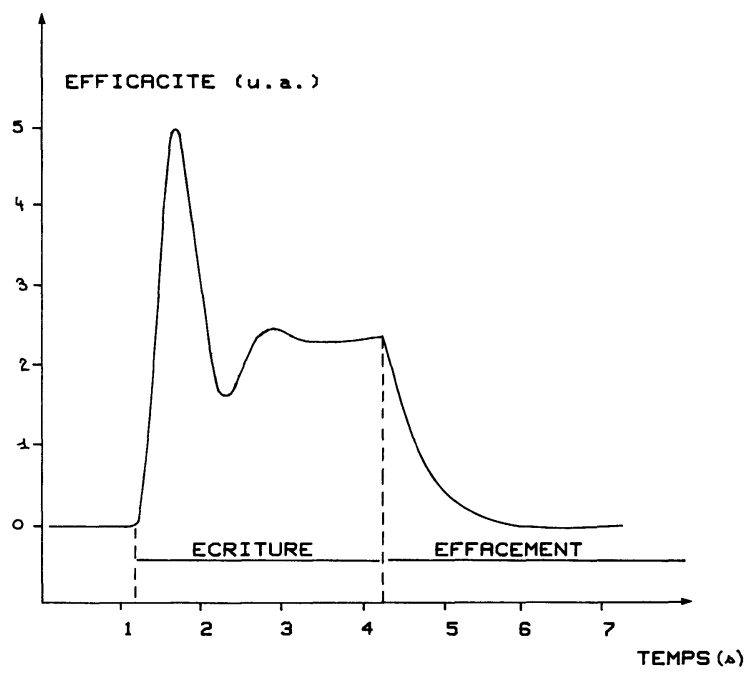

Fig. 6. - Evolution temporelle de l'efficacité de diffraction d'un réseau de faible modulation lors d'un cycle d'inscription-effacement sous champ électrique appliqué.

[Rise and decay of the diffraction efficiency under applied electric field.]

fonction du temps, illustre la cinétique d'établissement du champ de charge d'espace (Eq. (14)). Durant l'inscription du réseau, on observe bien une sur-oscillation [28]. Ses caractéristiques dépendent de la valeur du champ électrique appliqué $E_{0}$ (Eq. (15)). Lors de l'effacement, la décroissance est purement exponentielle avec une constante de temps dépendant également de $E_{0}$. L'influence de ce champ sur la cinétique de l'effet photoréfractif est illustrée par les figures 7 et 8 qui montrent l'effet du champ sur l'inverse de la constante de temps $(1 / \tau)$ et sur la fréquence d'oscillation $(\omega)$ [29].

Le phénomène de sur-oscillation est mis à profit dans certaines applications (par exemple pour le couplage d'ondes). Il est en effet possible d'introduire une résonance dans l'équation (14) en ajoutant

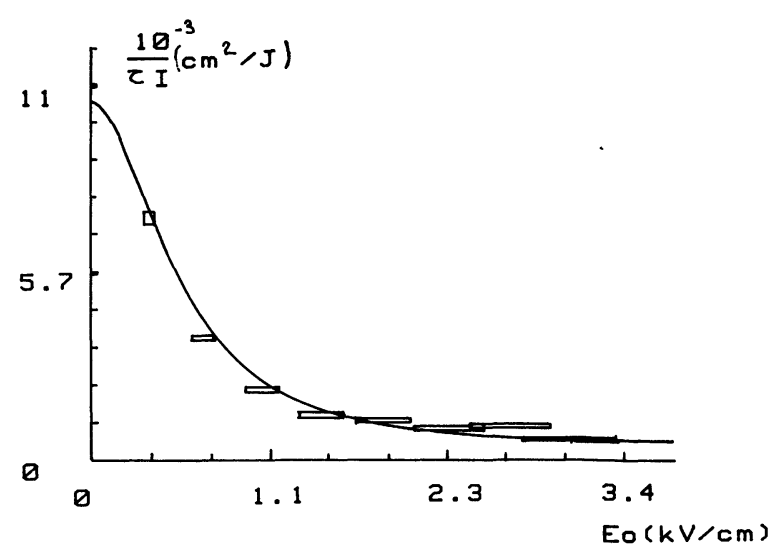

Fig. 7. - Influence du champ électrique appliqué sur la constante de temps d'évolution du réseau photoréfractif (— prévisions du modèle, $\square$ points expérimentaux).

[Dependence of the grating time constant upon applied electric field ( - theoretical $\square$ experimental data).]

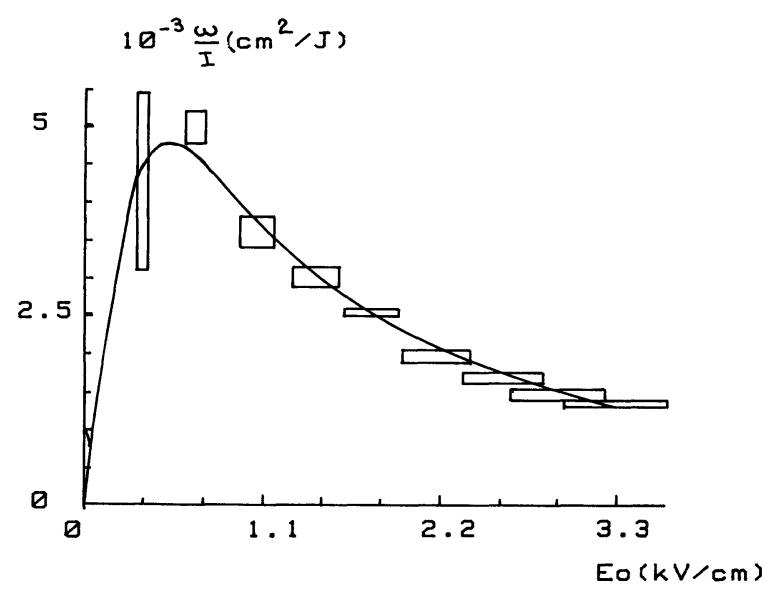

Fig. 8. - Influence du champ électrique appliqué sur la fréquence d'oscillation du réseau photoréfractif lors de l'enregistrement ( - prévisions du modèle, $\square$ points expérimentaux).

[Dependence of the grating oscillation frequency upon applied electric field ( - theoretical $\square$ experimental data).]

un terme oscillant à la fréquence $\omega$. Ce terme est obtenu en éclairant le cristal par une figure d'interférence mobile se déplaçant à la vitesse $v=\omega / \kappa_{\mathrm{R}}$ $[37,38]$. On peut aussi moduler $m$ à la fréquence $\omega[34]$, ou utiliser une modulation temporelle du champ électrique $E_{0}$ [39].

$E_{0}$ influe aussi sur la valeur du champ de charge d'espace à l'état stationnaire [40] (Eq. (12)), ainsi que le montrent les courbes de la figure 9. Cela a récemment permis d'obtenir des efficacités de diffraction voisines de $95 \%$ [41].

4.2 DÉTERMINATION DU SIGNE DES PORTEURS PAR COUPLAGE D'ONDES. - Des expériences de photocourant [22] montrent que selon le taux de dopage d'un cristal en ions aluminium, les porteurs majori- 


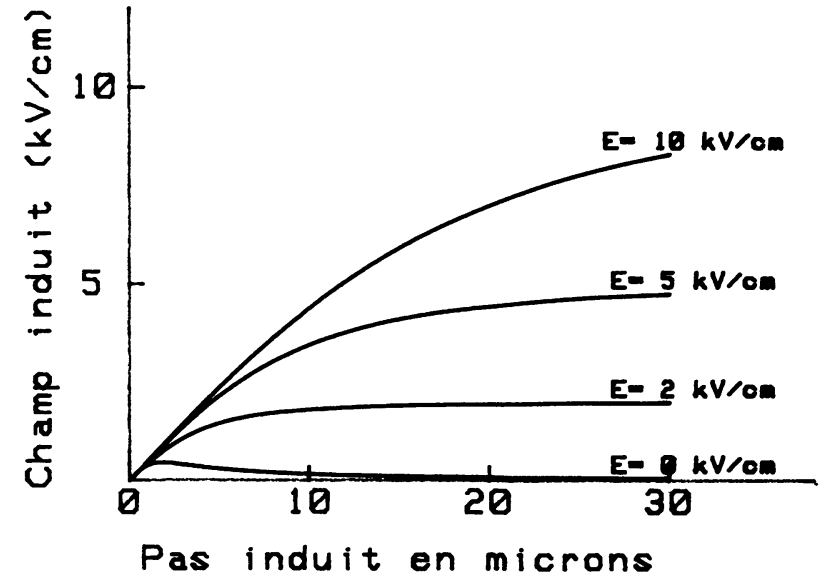

Fig. 9. - Influence du champ électrique appliqué sur la valeur en régime permanent du champ de charge d'espace.

[Electric field dependence of the steady state space charge field.]

taires sont des électrons (cristaux faiblement dopés) ou des trous (cristaux fortement dopés). Le modèle d'évolution temporelle de l'effet photoréfractif, précédemment développé, supposait que les porteurs de charge fussent des électrons. Un développement identique peut être effectué lorsque ces porteurs sont des trous. Toutes choses restant égales par ailleurs, l'équation d'évolution du réseau de champ de charge d'espace est déduite de l'équation (9) en remplaçant $i$ par $-i$. Cela conduit à changer le signe de $\sin \psi$ dans l'équation (13).

Le signe des porteurs libres ne peut être déterminé par l'observation de l'efficacité de diffraction qui dépend de $E_{1}^{2}$. Cette détermination peut être effectuée par des mesures de couplage d'ondes, sensibles au signe du déphasage $\psi$.

$\mathrm{La}$ théorie du couplage d'ondes [42] est dérivée des équations des ondes couplées [43]. Les conventions d'orientation étant celles de la figure 5, en sortie du cristal, l'intensité $I_{\mathrm{s}}(e)$ mesurée dans la direction du faisceau sonde vaut :

$$
I_{\mathrm{s}}(e)=\frac{I_{0}}{1+\frac{I_{\mathrm{p}}(0)}{I_{\mathrm{s}}(0)} e^{-G e}} e^{-\alpha e}
$$

et celle de $I_{\mathrm{p}}(e)$ mesurée dans la direction du faisceau pompe,

$$
I_{\mathrm{p}}(e)=\frac{I_{0}}{1+\frac{I_{\mathrm{s}}(0)}{I_{\mathrm{p}}(0)} e^{G e}} e^{-\alpha e}
$$

avec $I_{\mathrm{p}}(0)$ et $I_{\mathrm{s}}(0)$ les intensités des faisceaux pompe et sonde à l'entrée du cristal,

$-I_{0}=I_{\mathrm{p}}(0)+I_{\mathrm{s}}(0)$

$-e$ l'épaisseur du cristal ;
- $\alpha$ le coefficient d'absorption ;

- $G$ le gain par unité de longueur défini par:

$$
\begin{aligned}
G=\frac{\omega n^{3}}{2 c \cos \theta} E_{\mathrm{sc}} & r_{\mathrm{eff}} \sin \psi= \\
& =\frac{\omega}{2 c} \frac{n^{3}}{\cos \theta} E_{\mathrm{sc}}\left|r_{\mathrm{eff}}\right| \sin \psi^{\prime}
\end{aligned}
$$

où $r_{\text {eff }}$ représente le coefficient électro-optique effectif vu par les ondes pompe et sonde supposées ondes propres du milieu [44]. Lorsqu'aucun champ n'est appliqué au cristal, ces ondes propres sont circulaires et :

$$
r_{\text {eff }}=r_{41}\left(1+\sin ^{2} \theta\right) \cos ^{2} \theta .
$$

Le signe de $r_{\text {eff }}$ est donc celui de $r_{41}$ et ne peut être connu a priori. Par rapport à l'axe $\mathbf{k}^{\prime}$ (Fig. 5), dire que $r_{41}$ est positif signifie que l'indice selon l'axe $i^{\prime}$ devient :

$$
n-\frac{r_{41} E_{0} n^{3}}{2}<n
$$

si l'on applique, suivant l'axe $\mathbf{k}^{\prime}$, un champ $\mathbf{E}_{0}$. Dans ce cas, si les porteurs responsables de l'effet photoréfractif sont des électrons, $\psi=\psi^{\prime}=-\pi / 2$ et l'onde sonde est atténuée.

Pour connaître le signe des porteurs, il est donc nécessaire, en plus des expériences de couplage, de déterminer le signe de $r_{\text {eff }}$ par rapport à l'axe $\mathbf{k}^{\prime}$ considéré [44].

De telles expériences, prenant en compte la forte biréfringence circulaire des cristaux de BSO et de BGO, ont montré que l'effet photoréfractif résultait de porteurs de signes différents selon les échantillons [45].

\subsection{PARAMÈTRES CARACTÉRISTIQUES EN RÉGIME} CONTINU. - De même que l'étude du couplage d'onde permet une détermination du signe des porteurs responsables de l'effet photoréfractif, l'étude de la cinétique de l'efficacité de diffraction lors de cycles d'inscription-effacement du réseau, permet une mesure des paramètres du modèle. Lorsque la durée de l'illumination est grande devant le temps de recombinaison des porteurs de charge, la description de l'effet photoréfractif nécessite la connaissance de trois paramètres :

- $\tau_{\mathrm{DI}}$, la constante de temps de relaxation diélectrique sous illumination (photoconductivité),

- $\kappa$, l'inverse de la longueur de diffusion $L$ des porteurs de charge (liée au produit mobilité-temps de recombinaison),

- $k_{\mathrm{d}}$ l'inverse de la longueur d'écran de Debye $L_{\mathrm{d}}$ (liée à la densité de pièges).

Un autre paramètre important est la conductivité du cristal dans le noir. Elle définit la durée de mémorisation $\tau_{\mathrm{m}}$ et peut être mesurée en observant 
Tableau I. - Comparaison des paramètres photoréfractifs dans différents cristaux de BSO et de BGO.

\begin{tabular}{|c|c|c|c|c|}
\hline ECHANT ILLON & $\begin{array}{|cc|}\text { BGO } & 1 \\
\operatorname{Ref}[36,44]\end{array} \mid$ & 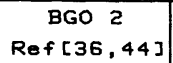 & $\begin{array}{|cc|}\text { BSO } & 1 \\
\operatorname{Ref}[36,44]\end{array}$ & $\begin{array}{l}\text { BSO } 2 \\
\text { Ref [35] }\end{array}$ \\
\hline $\begin{array}{l}\text { Vitesse de } \\
\text { tirage }(\mathrm{mm} h-1)\end{array}$ & 1,2 & 2,0 & & \\
\hline $\begin{array}{l}\text { Vitesse de } \\
\text { rotation(tours mm-1) }\end{array}$ & 20 & 20 & & \\
\hline DOPRGE & Non & Non & Non & Non \\
\hline $\begin{array}{l}\text { Absorption optique } \\
(514 \mathrm{~nm})(\mathrm{cm}-1)\end{array}$ & 1,45 & 0,93 & 1,67 & \\
\hline $\begin{array}{l}\text { Activité optique } \\
\text { à } 633 \mathrm{~nm}(\text { degré } \mathrm{mm}-1 \text { ) }\end{array}$ & $+20,8$ & $+20,7$ & $-22,8$ & \\
\hline $\begin{array}{l}\text { Longueur de } \\
\text { diffusion } L \text { en } N m\end{array}$ & $2,3 \pm 8 \%$ & $8,0 \pm 13 \%$ & $3,4 \pm 20 \%$ & 3 , \\
\hline $\begin{array}{l}\text { Longueur d'écran } \\
\text { de Debye ld en } \mu \mathrm{m}\end{array}$ & $0,29 \pm 33 \%$ & 0,27 & $0,10 \pm 20 \%$ & 0,08 \\
\hline $\begin{array}{l}\text { I mesuré } \\
\text { elect. }(\mu \mathrm{J} \mathrm{cm}-2)\end{array}$ & $3,7 \pm 20 \%$ & $0,3 \pm 20 \%$ & $1,0 \pm 2$ & 1,6 \\
\hline $\begin{array}{l}\text { Temps de mémorisation } \\
? \mathrm{~m}\end{array}$ & $=16 \mathrm{H}$ & $6 \mathrm{mn}$ & & \\
\hline $\begin{array}{l}\text { Signe des } \\
\text { porteurs libres }\end{array}$ & + & - & - & \\
\hline Temps de recombinaison & $22 \pm 5 \%$ & $56 \pm 7 \%$ & $550 \pm 20 \%$ & \\
\hline
\end{tabular}

le temps de décroissance dans le noir d'un réseau photoinduit. Pour déterminer les trois premiers paramètres, on peut choisir de faire varier soit le pas du réseau photoinduit [35] soit l'amplitude du champ électrique appliqué [36].

Certains parmi ces paramètres peuvent aussi être déterminés par l'étude de la biréfringence induite [46].

Le tableau I présente les résultats obtenus pour quelques cristaux photoréfractifs. Les BGO ont été cristallisés au laboratoire de chimie du solide du CNRS (Bordeaux), les BSO par Crystal Technology. Pour deux échantillons de conditions de cristallogénèse voisines (BGO 1 et $\mathrm{BGO} 2$ ), les longueurs de diffusion et les constantes de relaxation diélectrique diffèrent de façon significative. La longueur de diffusion la plus grande $(8 \mu \mathrm{m})$ correspond à une migration d'électrons et la plus courte $(2,3 \mu \mathrm{m})$ à une migration de trous. Notons également la différence importante entre les durées de mémorisation dans le noir. Par ailleurs, les valeurs des longueurs d'écran de Debye montrent que la densité $N_{\mathrm{A}}$ dans ces cristaux de BGO $\left(7,8 \times 10^{14} \mathrm{~cm}^{-3}\right)$ est environ dix fois plus faible que dans les échantillons de BSO. $\left(N_{\mathrm{A}}\right.$ intervient par son carré dans l'efficacité de diffraction à grand pas de réseau photoinduit et à fort champ appliqué).

Dans ce tableau ont été reportées à titre de comparaison, les valeurs du temps de recombinaison obtenues à partir de la cinétique du photocourant. Le comportement d'autres échantillons ne peut être décrit par le modèle. Il devient alors nécessaire d'envisager la participation simultanée d'électrons et de trous à l'effet photoréfractif $[47,48]$ ou encore l'existence de plusieurs types de pièges dans la bande interdite $[48,49]$. On en trouve une illustration dans les expériences d'effet photoréfractif en régime impulsionnel $[31,50]$.

\section{Excitation impulsionnelle.}

Le développement théorique de la partie précédente suppose que la durée de l'illumination et le temps d'établissement de l'effet photoréfractif sont longs devant le temps de recombinaison des porteurs excités. Lorsque le réseau est produit par l'interférence de deux impulsions lumineuses suffisamment brèves $[30,31,50]$, cela n'est pas toujours vérifié. On peut connaître le temps de recombinaison des porteurs de charge en étudiant la cinétique du photocourant [22, 51-53] $\left(n_{0}(t)\right.$ dans l'Eq. (6)).

Les enregistrements de la figure 10 montrent l'évolution temporelle du photocourant d'un cristal de BSO uniformément illuminé par une impulsion de durée $\tau_{\mathrm{p}}=3 \mathrm{~ns}(\lambda=532 \mathrm{~nm})$. La décroissance du photocourant présente deux constantes de temps très différentes, $\tau_{1}=50 \mathrm{~ns}$ (Fig. 10a) et $\tau_{2}=10 \mu \mathrm{s}$ (Fig. 10b) [31, 54]. La recombinaison des porteurs de charge mettrait donc en jeu deux types de pièges. La partie rapide de la décroissance est attribuée à un piégeage des porteurs dans des centres peu profonds (permettant leur réexcitation thermique), sa partie lente correspond à leur recombinaison dans les pièges profonds (donnant lieu à l'effet mémoire). Ce dernier processus est celui décrit dans le précédent paragraphe.

Le piégeage dans les centres peu profonds est démontré dans une expérience utilisant deux excitations à des longueurs d'ondes très différentes. Aucun photocourant n'est observé lorsque le cristal est 


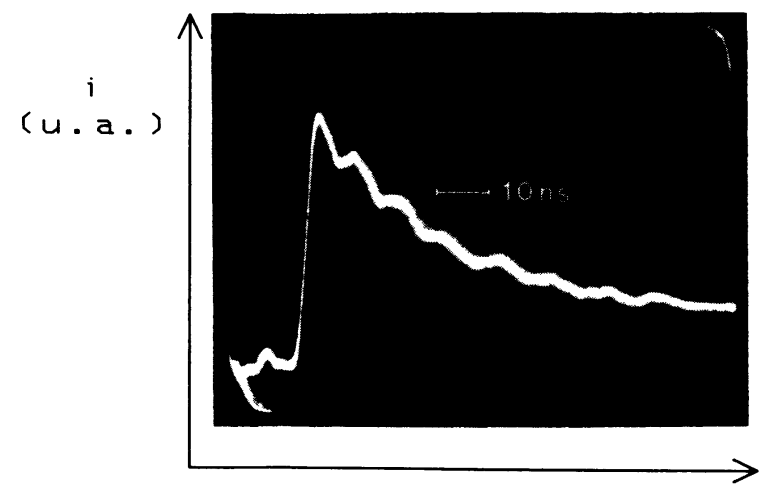

a)

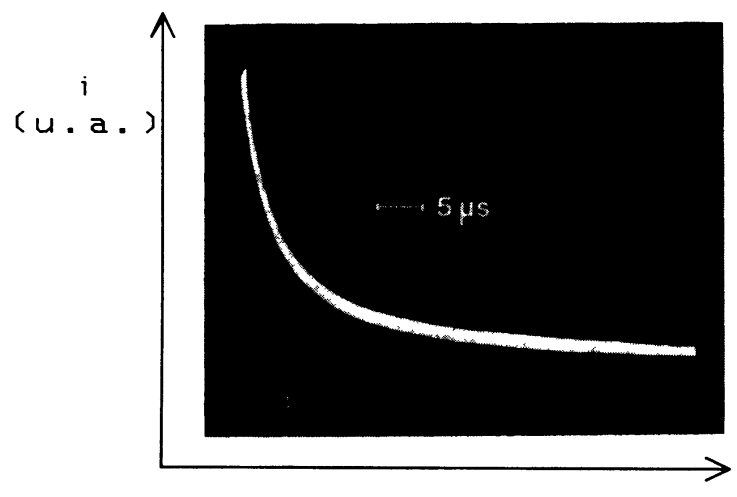

b)

Fig. 10. - Evolution temporelle du photocourant créé par une impulsion lumineuse de durée $3 \mathrm{~ns}$ et de longueur d'onde $532 \mathrm{~nm}$; l'échelle des temps est $10 \mathrm{~ns}$ par carreau (a) et $5 \mu$ s par carreau (b).

[Temporal behaviour of the photocurrent induced by the optical pulse (duration $3 \mathrm{~ns}$, wavelength $532 \mathrm{~nm}$ ) ; (a) time scale $10 \mathrm{~ns}$ per div (b) time scale $5 \mu$ s per div.]

illuminé par un faisceau laser à la longueur d'onde $\lambda=1,06 \mu \mathrm{m}$ (pour laquelle le BSO est transparent). Si par contre, cette impulsion infrarouge éclaire le cristal immédiatement après une impulsion de longueur d'onde à $0,53 \mu \mathrm{m}$, on observe deux pics de photocourant séparés du même intervalle de temps que les impulsions excitatrices (Fig. 11) [50]. Si cet intervalle est trop important ( $\geqslant 1 \mathrm{~ms}$ ), le second pic de photocourant disparaît ; on montre ainsi que le piégeage dans les centres peu profonds est temporaire. Pour certains cristaux de BSO, le temps de piégeage rapide est plus court que la durée de l'impulsion [50, 54, 55].

Pour rendre compte de ces résultats, le modèle doit être développé en remplaçant les équations (1)(4) par celles prenant en compte les divers processus d'excitation et de recombinaison [31] (Fig. 11). Le modèle rend alors bien compte de l'évolution du photocourant observée sur la figure 10. Il permet une détermination de la mobilité des porteurs dans

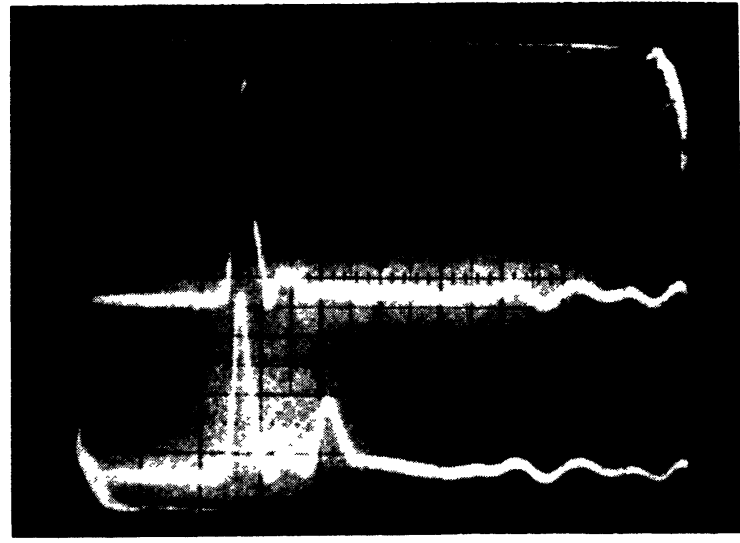

Fig. 11. - Mise en évidence d'une recombinaison temporaire dans des pièges peu profonds : le pic de photocourant produit par l'impulsion à $532 \mathrm{~nm}$ (trace supérieure) est suivi d'un second pic dû à l'impulsion à $1,06 \mu \mathrm{m}$ (trace inférieure) lorsque le délai entre ces impulsions n'est pas trop élevé (ici $30 \mathrm{~ns}$ ).

[Upper curve : photocurrent induced by the pulse at $532 \mathrm{~nm}$ in a BSO crystal (The trapping time is shorter than the pulse duration). Lower curve : photocurrent induced by both green and infrared pulses with a delay a $30 \mathrm{~ns}$. The BSO crystal becomes conductive at $\lambda=1,06 \mu \mathrm{m}$.]

la bande de conduction. La valeur $\mu=$ $3 \mathrm{~cm}^{2} \mathrm{~V}^{-1} \mathrm{~s}^{-1}$ déterminée ainsi pour l'échantillon considéré est environ cent fois plus importante que celle couramment utilisée dans la littérature [22] : la présence des pièges peu profonds se traduit par une réduction apparente de la mobilité des porteurs dans les expériences en régime quasi continu.

L'étude du photocourant montre que pour cet échantillon, les processus de recombinaison se produisent après le passage de l'impulsion $\left(\tau_{\mathrm{p}}<\tau_{1}<\right.$ $\left.\tau_{2}\right)$. La détermination du champ de charge d'espace résultant de l'interférence de deux impulsions lumineuses s'effectue en deux temps. Durant l'illumination, on considère tout d'abord qu'il n'y a ni piégeage ni recombinaison: seuls les processus de photo-excitation et de migration des charges participent à la construction du champ de charge d'espace. Ensuite, après l'impulsion, seuls les processus de migration et de recombinaison (éventuellement multiples) sont à prendre en compte dans les équations. Les conditions initiales sont alors définies par l'état du système à $t=\tau_{\mathrm{p}}$.

Le champ de charge d'espace pendant la durée de l'impulsion (supposée rectangulaire) est calculé à partir des équations (1) à (6) modifiées et en tenant compte de la dépendance temporelle de la densité moyenne de porteurs $n_{0}(t)=s I_{0} N_{\mathrm{D}} t$ [31]. Considérant toujours le cas des faibles modulations $(m \ll 1)$, l'équation d'évolution temporelle du champ de charge d'espace $E_{1}$ durant l'illumination $\left(t<\tau_{\mathrm{p}}\right)$ 
s'écrit alors :

$$
\begin{aligned}
\frac{\partial E_{1}}{\partial t}+E_{1}\left(\frac{t}{\tau_{\mathrm{p}} \tau_{\mathrm{DI}}}+\frac{1}{\tau_{\mathrm{D}}}\right. & \left.-\frac{i}{\tau_{\mathrm{E}}}\right)+ \\
& +m \frac{E_{0}+i E_{\mathrm{D}}}{\tau_{\mathrm{DI}}} \cdot \frac{t}{\tau_{\mathrm{p}}}=0
\end{aligned}
$$

Elle ressemble à l'équation (10), mais ses coefficients dépendent du temps. Les constantes $\tau_{\mathrm{DI}}$,
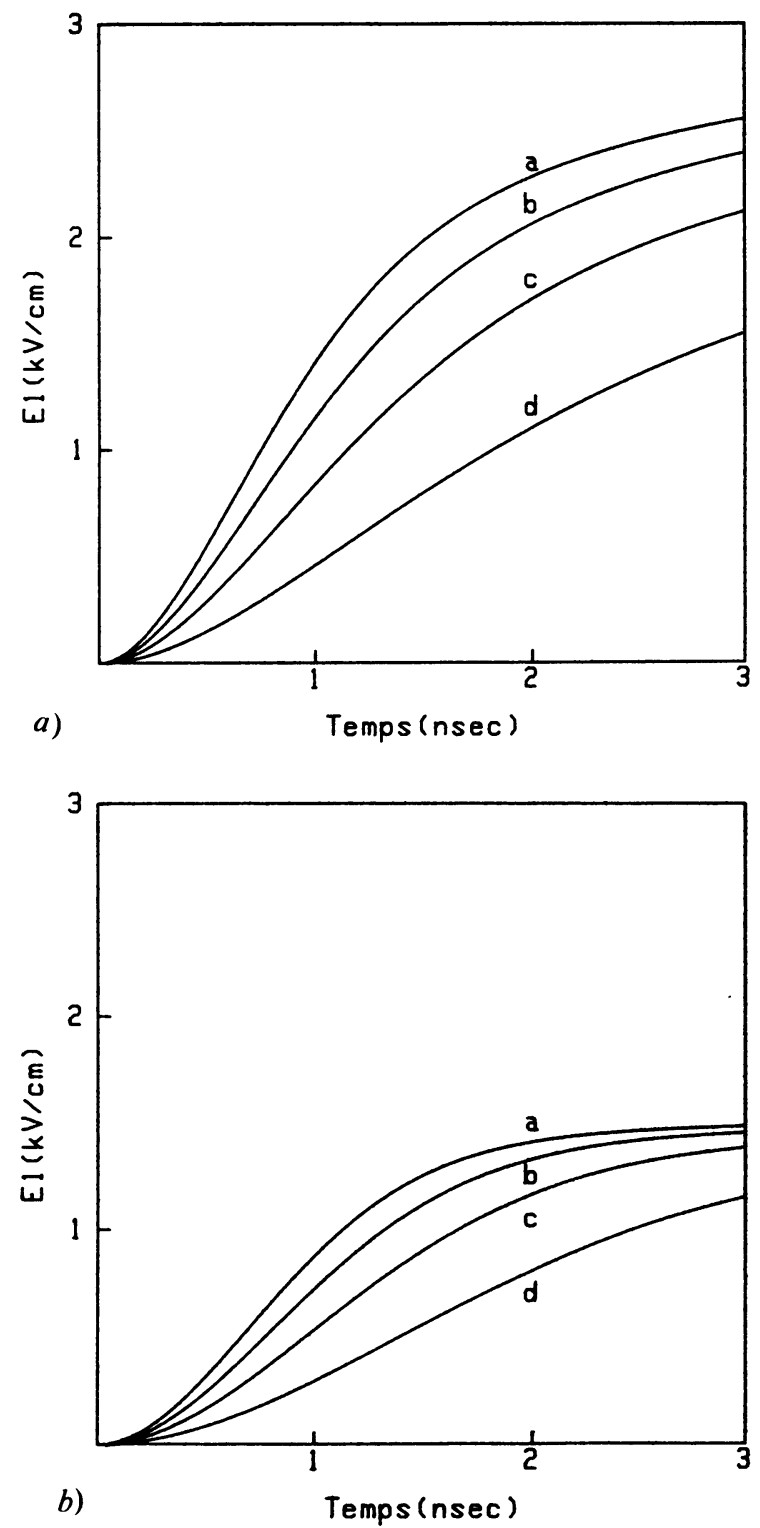

Fig. 12. - Evolution de l'amplitude du champ de charge d'espace calculée pour différentes valeurs de l'énergie absorbée $I_{0}$ et deux valeurs du pas $\Lambda$ du réseau induit : 12 a) $\Lambda=0,5 \mu \mathrm{m}$; 12 b) $\Lambda=1 \mu \mathrm{m}$. Pour les deux figures $I_{0}=20 \mathrm{~mJ} . \mathrm{cm}^{-2}$ (a), $15 \mathrm{~mJ} . \mathrm{cm}^{-2}$ (b), $10 \mathrm{~mJ} . \mathrm{cm}^{-2}$ (c), $5 \mathrm{~mJ} . \mathrm{cm}^{-2}(\mathrm{~d})$.

[Space charge field amplitude variation as a function of time during the optical pulse for different grating periods : 12 a) $\Lambda=0,5 \mu \mathrm{m}, 12 \mathrm{~b}) \Lambda=1 \mu \mathrm{m}$, and various energies : $I_{0}=20 \mathrm{~mJ} . \mathrm{cm}^{-2} \quad$ (a), $I_{0}=15 \mathrm{~mJ} . \mathrm{cm}^{-2} \quad$ (b), $I_{0}=$ $10 \mathrm{~mJ} . \mathrm{cm}^{-2}$ (c), $I_{0}=5 \mathrm{~mJ} . \mathrm{cm}^{-2}$ (d).]
$\tau_{\mathrm{D}}$ et $\tau_{\mathrm{E}}$ sont définies par les relations (9). $\tau_{\mathrm{DI}}$ représente cependant ici, la valeur de la constante de temps diélectrique à la fin de l'impulsion. La figure 12 montre l'évolution temporelle, pendant la durée de l'impulsion et sans champ appliqué $\left(E_{0}=0\right)$, du champ de charge d'espace $E_{1}$ calculé à partir de (19) pour différentes valeurs du pas du réseau photoinduit et de l'énergie optique absorbée. Pour ces tracés ; on a utilisé la valeur de la mobilité $\mu$ mesurée précédemment $\left(3 \mathrm{~cm}^{2} \mathrm{~V}^{-1} \mathrm{~s}^{-1}\right)$. Ainsi, l'effet photoréfractif se construit pendant l'impulsion et peut même atteindre sa valeur maximale en moins de $3 \mathrm{~ns}$ (Fig. $12 \mathrm{~b}, \Lambda=1 \mu \mathrm{m}, I_{0}>15 \mathrm{~mJ} / \mathrm{cm}^{-2}$ ). Ce résultat théorique est illustré par la figure 13. Il montre un relevé expérimental de l'efficacité de diffraction en fonction du temps en le comparant à

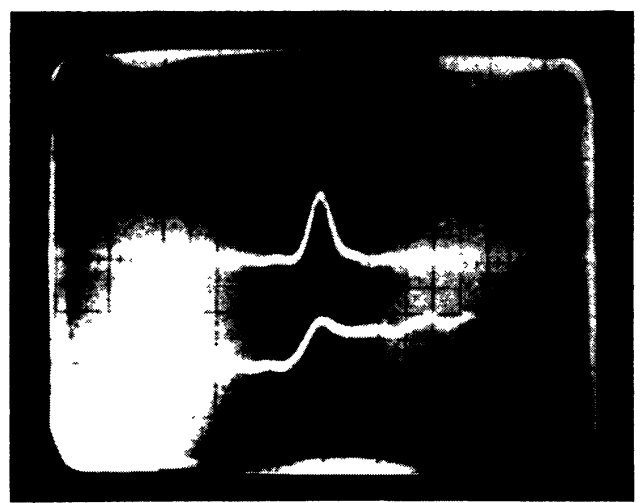

Fig. 13. - Enregistrement de l'évolution temporelle du faisceau diffracté (trace inférieure) résultant de l'enregistrement d'un réseau photoréfractif au moyen d'impulsions de durée 3 ns (trace supérieure) ; l'échelle de temps est de 20 ns par carreau.

[Upper curve : pulse shape of the incident writing beams. Lower curve : hologram recording kinetics with a time scale : $20 \mathrm{~ns}$ per div.]

celui de l'impulsion excitatrice. Les courbes de la figure 14 montrent, pour différentes valeurs du pas du réseau induit, l'influence de l'énergie lumineuse sur la valeur du champ de charge d'espace à la fin de l'impulsion. Pour des valeurs de l'énergie lumineuse $I_{0}$ inférieures à $2 \mathrm{~mJ} / \mathrm{cm}^{2}$, le champ de charge d'espace $E_{1}$ croît linéairement avec $I_{0}$ puis sature lorsque l'on augmente l'intensité. Ce résultat est confirmé par l'évolution expérimentale de l'efficacité de diffraction de la figure 15.

A cause de la relation (4), le champ de charge d'espace $E_{1}$ présente toujours (en champ nul $\left.E_{0}=0\right)$, une avance de phase de $\pi / 2$ par rapport à la figure d'interférence (cas des porteurs de charge négatifs). Cela n'est en rien modifié par la prise en compte du second type de pièges. L'établissement de ce déphasage étant instantané par nature, la cinétique du transfert d'énergie par couplage d'ondes 


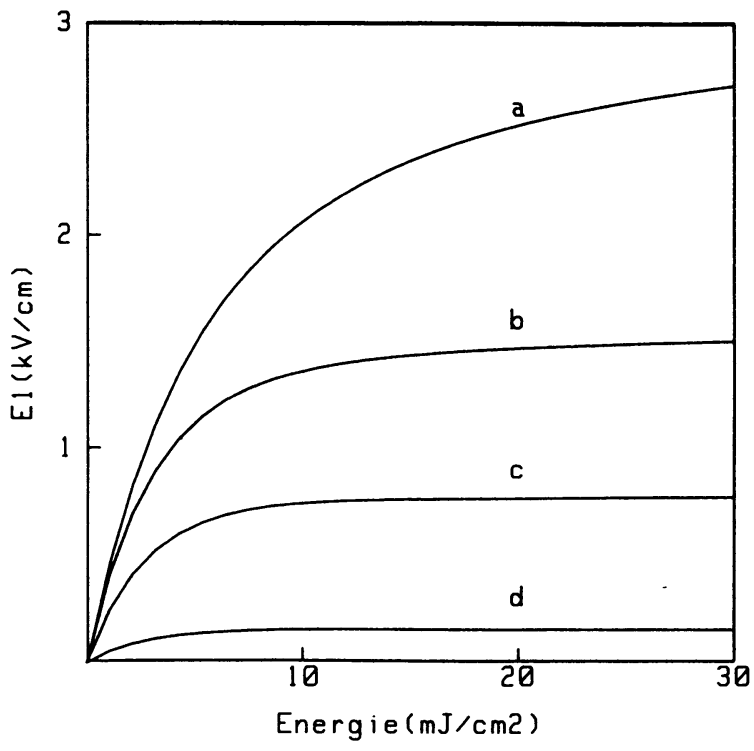

Fig. 14. - Valeur de l'amplitude du champ de charge d'espace calculée à la fin de l'illumination pour différentes valeurs du pas du réseau induit : $\Lambda=0,5 \mu \mathrm{m}$ (a), $1 \mu \mathrm{m}$ (b), $2 \mu \mathrm{m}$ (c), $10 \mu \mathrm{m}$ (d).

[Space charge field amplitude variation at the end of the optical pulse as a function of the energy for various grating periods $\Lambda=0,5 \mu \mathrm{m}$ (a), $1 \mu \mathrm{m}$ (b), $2 \mu \mathrm{m}$ (c), $10 \mu \mathrm{m}$ (d).]

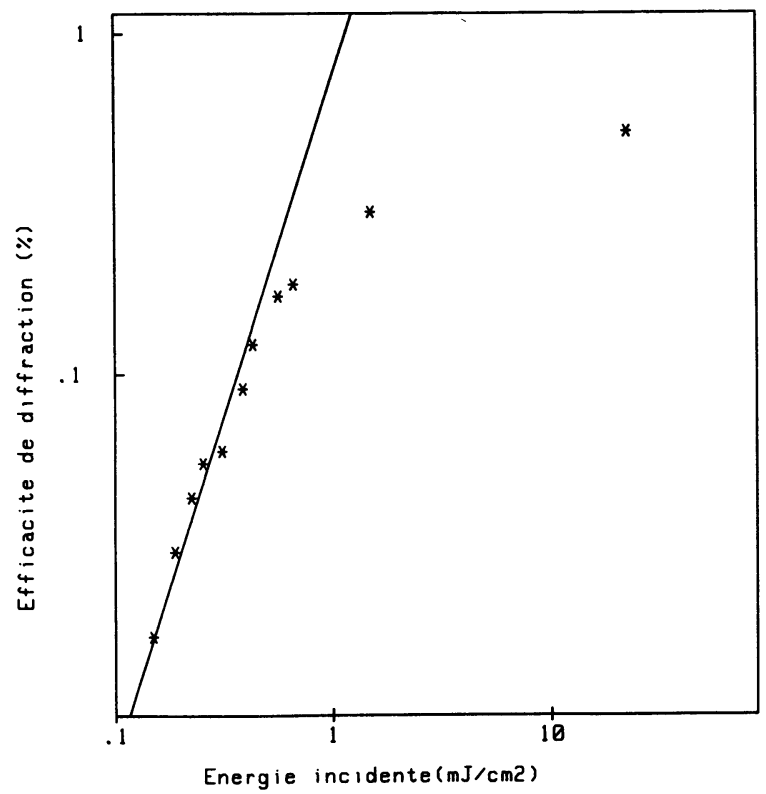

Fig. 15. - Influence de l'énergie incidente sur la valeur de l'efficacité de diffraction : $\Lambda=0,7 \mu \mathrm{m}$.

[Diffraction efficiency as a function of the recording energy : $\Lambda=0,7 \mu \mathrm{m}$.]

ne dépend donc que de l'évolution temporelle du champ de charge d'espace. En se plaçant dans des conditions expérimentales convenables $(\Lambda=$ $0,5 \mu \mathrm{m}, I_{0}=20 \mathrm{~mJ} \mathrm{~cm}^{-2}$ ), le transfert d'énergie par couplage d'ondes a pu être observé pendant les $3 \mathrm{~ns}$ de l'impulsion [56]. Ces expériences sont illustrées par la figure 16. Elle montre l'impulsion sonde transmise en l'absence du faisceau pompe (a) puis cette même impulsion en présence de ce faisceau (b). L'amplification $\gamma_{1}$ due au couplage d'ondes vaut alors 0,5 . Une rotation de $180^{\circ}$ du cristal autour de son axe $(\overline{1}, 1,0)$ conduit à une amplification $\gamma_{2}=$ 1,8 du signal sonde [56]. $\gamma$ est liée au gain $G$ (relation (11)) par $\gamma=\exp [\mathrm{Ge}]$. Dans les conditions expérimentales, et en remplaçant $E_{\mathrm{sc}}$ par la valeur $\frac{E_{1}\left(\tau_{\mathrm{p}}\right)}{m}=2,6 \mathrm{kV} \mathrm{cm}^{-1}$, la relation (16) prévoit les valeurs $\gamma_{1}=0,53$ et $\gamma_{2}=1,9$.

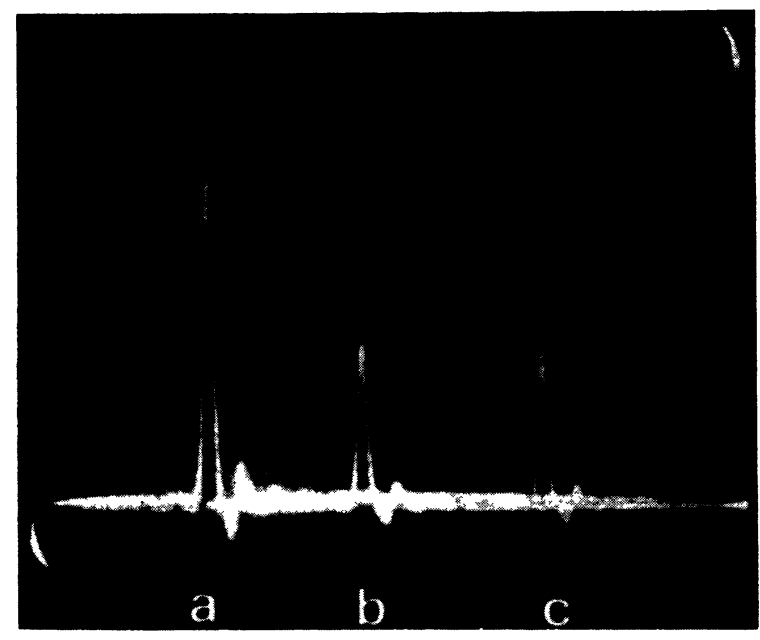

Fig. 16. - Transfert d'énergie par couplage d'ondes entre deux impulsions de durée $3 \mathrm{~ns}$ : faisceau sonde seul (a), faisceau sonde en présence de la pompe lors d'un tir unique (b), après plusieurs tirs (c).

[Energy transfer between two 3 ns optical pulses : (a) the probe beam only, (b) probe transmission in presence of the pump after a single shot, (c) probe transmission in presence of the pump after several shots.]

L'étude d'autres échantillons de BSO et de BGO [31], a révélé des comportements différents lorsque la constante de temps de recombinaison est plus courte que la durée de l'impulsion.

\section{Applications de l'effet photoréfractif et perspecti-} ves.

Les cristaux photoréfractifs de BSO et de BGO sont disponibles en grandes dimensions avec une très bonne qualité optique. Les premières utilisations pour l'enregistrement de réseaux holographiques de volume [6] ont mis en évidence leur grande sensibilité et la totale réversibilité du phénomène de modulation d'indice. Depuis lors, l'effet photoréfractif dans les cristaux de BSO (BGO) a été souvent mis à profit dans des expériences d'interférométrie dyna- 
mique [57], de traitement d'image [58-61], de déflexion de faisceaux laser [62], de conjugaison de phase et de cavités auto-oscillantes [63]. Des applications similaires ont été développées parallèlement avec d'autres types de cristaux photoréfractifs tels le titanate de baryum ou le niobate de potassium $[1,64]$.

Dans ce paragraphe, nous présentons brièvement quelques possibilités d'utilisation de l'effet photoréfractif dans le domaine du calcul optique. Selon les schémas retenus, les applications potentielles sont variées, tant en ce qui concerne la réalisation optique de mémoires associatives [13-15, 65, 66] d'opérations logiques élémentaires [7] que d'interconnexions reconfigurables $[67,68]$.

6.1 APplication du COUPLAGE DeUX ONDES A LA LOGIQUE OPTIQUE. - Le caractère non local de l'effet photoréfractif (déphasage spatial $\psi$ du réseau d'indice par rapport à la figure d'interférence) permet le transfert d'énergie entre les ondes qui se propagent dans le cristal. Lorsque le gain $G$ est élevé, ce phénomène peut être mis à profit pour la réalisation d'opérations logiques en vue du calcul optique numérique et parallèle. L'équation (18) montre qu'un fort gain requiert un déphasage $\psi$ voisin $\pi / 2$ et une valeur élevée du produit $r_{\mathrm{eff}} E_{\mathrm{sc}}$. Pour le BSO (BGO), $r_{\text {eff }}$ étant faible $\left(r_{41}=3,4-\right.$ $5 \mathrm{pm} . \mathrm{V}^{-1}$ [69-71]) il est nécessaire d'appliquer un champ électrique externe ce qui a cependant pour effet de réduire la valeur du déphasage. Pour contourner cette difficulté, il suffit de produire un léger décalage de fréquence sur l'un des faisceaux $[37,38]$. Ce décalage est ajusté de façon à ce que le réseau d'indice photoinduit reste en permanence déphasé de $\pi / 2$ par rapport au réseau d'illumination qui se déplace à vitesse constante. Des valeurs de $G$ de l'ordre de 8 à $12 \mathrm{~cm}^{-1}$ ont ainsi été observées expérimentalement [38]. Pour le titanate de baryum, étant donnée la forte valeur de son coefficient électro-optique $\left(r_{12}=800 \mathrm{pm} . \mathrm{V}^{-1}\right)$, ces valeurs de gain sont obtenues lors de l'enregistrement par diffusion du réseau de charge d'espace [64]. C'est avec ce matériau photoréfractif que les opérations logiques «OU, NON-OU, ET » ont été démontrées en régime d'excitation continue [7]. A titre d'illustration, nous donnons le principe conduisant à l'opération « NON-OU » (Fig. 17).

$$
\begin{aligned}
& \text { Notant } r=\frac{I_{\mathrm{s}}(0)}{I_{\mathrm{p}}(0)} \text {, le gain relatif } \gamma \mathrm{s} \text { 'écrit : } \\
& \gamma=\frac{I_{\mathrm{p}}(e) \text { [en présence de signal ] }}{I_{\mathrm{p}}(e) \text { [en absence de signal] }}=\frac{1+r}{1+r e^{G e}} .
\end{aligned}
$$

Il y a déplétion de la pompe en présence de signal lorsque $r e^{G e} \gg 1$, et alors :

$$
\gamma=\left(1+\frac{1}{r}\right) e^{-G e} .
$$

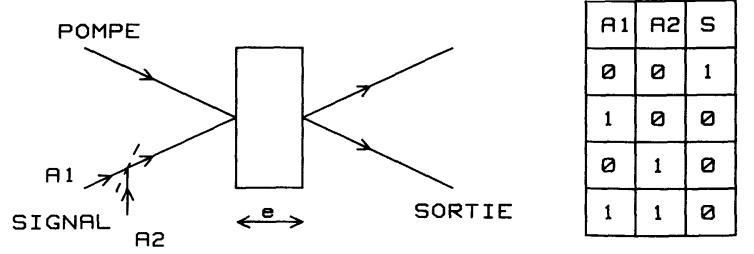

Fig. 17. - Principe de réalisation d'une opération logique NON-OU par transfert d'énergie dans un cristal photoréfractif.

[Optical NOR Gate implementation using a photorefractif crystal.]

Pour un cristal tel que $e^{G e} \cong 4000$ (ce qui avec les valeurs de $G$ mentionnées précédemment correspond à un cristal ayant une épaisseur de l'ordre du centimètre), ce phénomène de déplétion de la pompe est à la base de l'opération logique "NON-OU». Lorsque les deux entrées $A_{1}$ et $A_{2}$ ont une intensité faible (état logique 0 ), $r e^{G e} \ll 1$ et $r \ll 1$ et l'on a donc $\gamma=1$ : le signal de sortie $S$ est maximum (état logique 1). Lorsque l'une des deux (ou les deux) entrées ont une forte intensité (état 1), la valeur de $r \geqslant 1$ conduit à la déplétion de la pompe : le signal de sortie $S$ est faible (état 0 ).

Des composants logiques fonctionnant sur ce principe présentent les deux avantages fondamentaux suivants :

- les intensités des faisceaux d'entrées, de pompe et de sortie, sont du même ordre de grandeur;

- les longueurs d'ondes de tous ces signaux sont identiques.

Les entrées et les sorties de ces composants sont donc totalement compatibles et permettent la réalisation de n'importe quel circuit logique optique.

\subsection{APPLICATION AUX INTERCONNEXIONS DYNAMI-} QUES ET AUX MÉMOIRES ASSOCIATIVES. - L'adressage d'un faisceau élémentaire vers une direction quelconque de l'espace peut être obtenu par diffraction sur un réseau d'indice de pas variable. Cela est réalisé en modifiant la longueur d'onde des faisceaux qui interfèrent (faisceaux de contrôle) pour enregistrer ce réseau dans un matériau photosensible [16]. Cette méthode est analogue à une déflexion acoustooptique mais n'est pas soumise aux limitations liées à la faible vitesse de propagation de l'onde acoustique. La modification de l'onde optique stationnaire est en effet instantanée dans tout le champ d'interférence. La notion de temps de transit n'intervenant pas, le nombre de directions adressables est élevé.

La sélection de la longueur d'onde de contrôle pouvant être effectuée en des temps voisins de la nanoseconde [72], la vitesse de déflexion sera en général imposée par la constante de temps d'inscription du réseau d'indice dans le matériau.

Pour obtenir de grandes valeurs de déflexion, il 
faut pouvoir faire varier la fréquence spatiale du réseau photoinduit dans une gamme étendue. D'autre part l'obtention d'une efficacité de diffraction élevée requiert l'utilisation d'un réseau de volume avec lequel le faisceau à adresser doit interagir sous l'angle de Bragg. Pour satisfaire simultanément ces deux conditions, une solution consiste à modifier l'orientation du réseau en synchronisme avec la variation de son pas (Fig. 18a). Pour cela (Fig. 18b) on procède de la façon suivante : les faisceaux de contrôle interagissent avec une
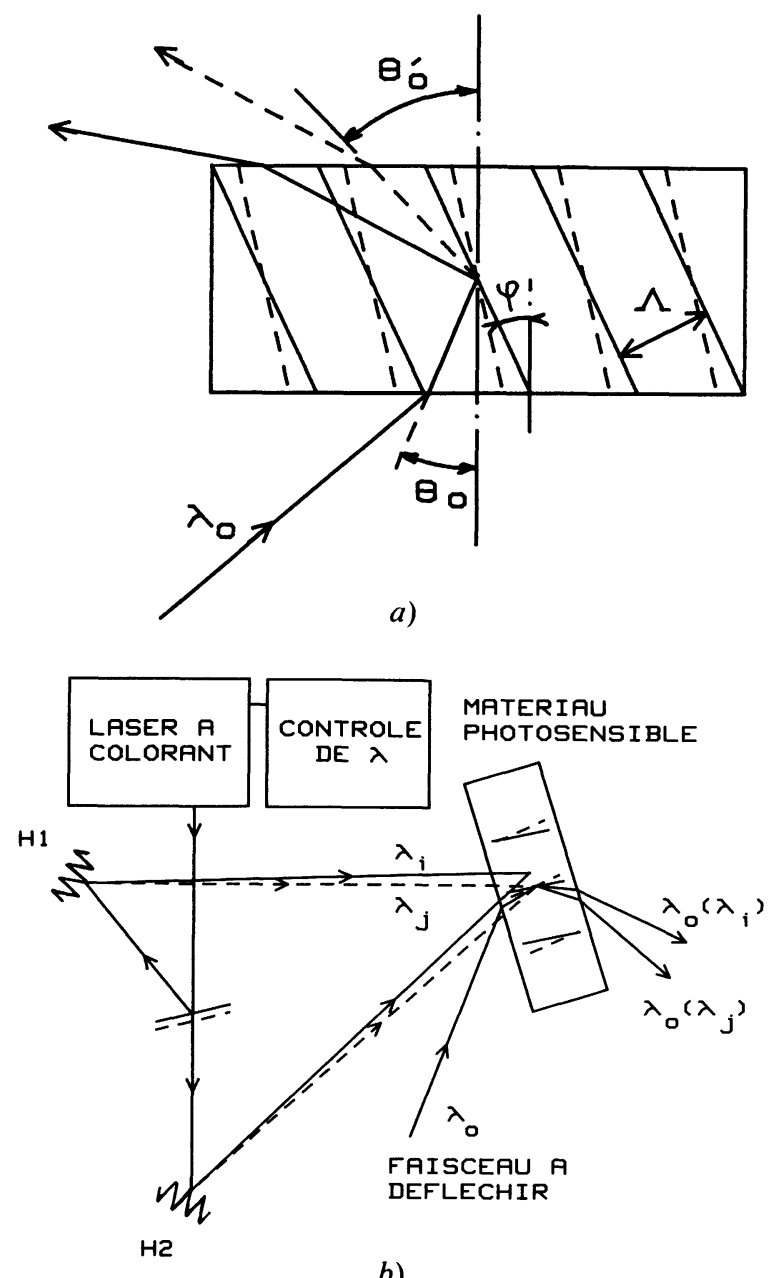

b)

Fig. 18. - a) Déflexion d'un faisceau lumineux par un réseau de volume reconfigurable: les plans des strates sont représentés pour deux pas $\Lambda$ et deux orientations $\varphi$ différentes. b) Principe de l'enregistrement du réseau dynamique à partir d'un faisceau à longueur d'onde variable.

(a) Laser beam steering by a dynamic volume hologram : grating is shown for two values of period $\Lambda$ and slant angle $\varphi$. b) Dynamic hologram recording using tunable wavelength writing beams.] structure dispersive fixe (réseaux $H_{1}$ et $H_{2}$, Fig. 18b). La modification de leur longueur d'onde produit alors une variation non symétrique de leurs directions d'incidence, d'où une modification simultanée de la fréquence spatiale et de l'orientation du réseau d'indice.

Un balayage en longueur d'onde de $27 \mathrm{~nm}$ a permis de défléchir un faisceau de lumière visible $(\lambda=633 \mathrm{~nm})$ sur plus de $15^{\circ}$. Etant donnée la divergence naturelle de ce faisceau, cela correspond à l'adressage de plus de 500 points sur une même ligne. L'adressage vers toute direction à l'intérieur d'un angle d'espace de $15^{\circ} \times 8^{\circ}$ a aussi été démontré expérimentalement [68]. L'utilisation de cristaux de BSO comme matériau photosensible réversible rend ces interconnexions reprogrammables.

La possibilité d'un enregistrement en volume dans les matériaux photoréfractifs permet un nombre élévé $N$ d'interconnexions simultanées $(N \cong$ Volume $/ \lambda^{3}$ ) et le comportement non linéaire de ces cristaux pourrait être utilisé pour simuler le fonctionnement de réseaux neuronaux. Ces propriétés sont à l'origine de récentes propositions de réalisation optiques de mémoires associatives [13-15, 66]. L'étude de ces réseaux dits neuronaux [73], se développe activement en raison, d'une part de la difficulté persistante à résoudre certains problèmes de reconnaissance d'images et d'autre part, des possibilités d'apprentissage que présentent de tels systèmes. Leur réalisation nécessite d'une part une opération non linéaire (seuil par exemple) [74] et d'autre part un système d'interconnexion reconfigurable [75]. On peut montrer qu'un hologramme est une forme de mémoire associative si on lui adjoint une non-linéarité. Il combine en effet la possibilité de corréler deux objets et la capacité de mémoriser ces objets.

6.3 Perspectives. - Les exemples précédents ne portent que sur des opérations élémentaires. Elles ne deviendront réellement attrayantes que lorsque ces éléments auront été assemblés de façon à effectuer un grand nombre d'opérations logiques, d'interconnexions ou de mémorisations. Cela nécessite une meilleure connaissance des matériaux photoréfractifs (propriétés physiques et photoréfractives) ainsi qu'un contrôle de leur cristallogénèse. Plusieurs équipes y travaillent dans le monde. La mise en évidence d'effets photoréfractifs dans les semiconducteurs (GaAs:Cr, InP:Fe [3] et GaAs non dopé [2]) avec une sensibilité apparemment plus forte encore que dans le BSO [76], permet d'envisager ce type de traitement optique dans le proche infrarouge. 


\section{Bibliographie}

[1] Gunter, P., Phys. Rep. 93 (1983) 200.

[2] Klein, M. B., Opt. Lett. 9 (1984) 350.

[3] Glass, A. M., Johnson, A. M., Olson, D. H., Simpson, W., Ballman, A. A., Appl. Phys. Lett. 44 (1984) 948.

[4] Huignard, J. P., Roosen, G., Proceedings in Physics 7, «Non-linear optics: Materials and devices », C. Flytzanis, J. L. Oudar, eds, (Springer Verlag) 1986, p. 128.

[5] Huignard, J. P., Herriau, J. P., Appl. Phys. Lett. 44 (1984) 948.

[6] Huignard, J. P., Micheron, F., Appl. Phys. Lett. 29 (1976) 591.

[7] Fainman, Y., Guest, C. C., Lee, S. H., Appl. Opt. 25 (1986) 1598.

[8] Nisenson, P., Iwasa, S., Appl. Opt. 11 (1972) 2760.

[9] Kwong, S. K., Rakulit, G. A., Yariv, A., Appl. Phys. Lett. 48 (1986) 201.

[10] Feinberg, J., Hellwarth, R. W., Opt. Lett. 5 (1980) 519 ; 6 (1985) 257.

[11] Rajbenbach, H., Huignard, J. P., Opt. Lett. 10 (1985) 137.

[12] Yeh, P., J. Opt. Soc. Am. B, 2 (1985) 1924.

[13] ANDERSon, D. Z., Opt. Lett. 11 (1986) 56.

[14] Soffer, B. H., DunNing, G. J., OWechKo, Y., MAROM, E., Opt. Lett. 11 (1986) 118.

[15] Yariv, A., Kwong, S. K., Opt. Lett. 11 (1986) 186.

[16] Sincerbox, G. T., Roosen, G., Appl. Opt. 22 (1983) 690.

[17] Pauliat, G., Herriau, J. P., Delboulbé, A., Roosen, G., Huignard, J. P., J. Opt. Soc. Am. B 3 (1986) 306.

[18] Abrahams, S. C., Jamieson, P. B., Bernstein, J. L., J. Chem. Phys. 47 (1967) 4034.

[19] Ballman, A. A., J. Cryst. Growth 1 (1967) 37.

[20] BASSAT, J. M., Thèse D.I., Univ. Bordeaux I, n 38 , 1986.

[21] Oberschmid, R., Phys. Statu. Solidi (a) 89 (1985) 263.

[22] Hou, S. L., Lauer, R. B., Aldrich, R. E., J. Appl. Phys. 44 (1973) 2652.

[23] Lauer, R. B., J. Appl. Phys. 42 (1971) 2147.

[24] Zielinger, J. P., Pohoryles, B., Balland, J. C., Grass, J. G., Coret, A., J. Appl. Phys. 57 (1985) 293.

[25] Tuninermans, C. W. M., Blasse, G., J. Solid State Chem. 52 (1984) 222.

[26] Burattini, E., Capuccio, G., Grandolfo, M., Vecchia, P., EfendieV, Sh. M., J. Opt. Soc. Am. Lett. 73 (1982) 495.

[27] Feinberg, J., Heiman, D., Tanguay, A. R., Hellwarth, R. W., J. Appl. Phys. 51 (1980) 1297.

[28] Kukhtarev, N. V., Sov. Tech. Phys. Lett. 2 (1976) 438.

[29] Jonathan, J. M. C., Hellwarth, R. W., Roosen, G., IEEE J. Quantum Electron. 22 (1986) 1936 ; J. Opt. Soc. Am. A 1 (1984) 1245.

[30] Hermann, J. P., Herriau, J. P., Huignard, J. P., Appl. Opt. 20 (1981) 2173.
[31] Le SAUX, G., Brun, A., soumis à IEEE J. Quantum Electron.

[32] YARIV, A., Quantum Electronics (John Wiler and Sons, N.Y.) p. 327.

[33] Valley, G. C., IEEE, J. Quantum Electron. QE 19 (1983) 1637.

[34] Pauliat, G., Thèse D. en Sciences, Univ. Paris-Sud, $\mathrm{n}^{\circ} 140,1986$.

[35] Mullen, R. A., Hellwarth, R. W., J. Appl. Phys. 58 (1985) 40.

[36] Pauliat, G., Jonathan, J. M. C., Allain, M., Launay, J. C., Roosen, G., Opt. Commun. 59 (1986) 266.

[37] Valley, G. C., J. Opt. Soc. Am. B 1 (1984) 868.

[38] Refregier, Ph., Solymar, L., Rajbenbach, H., Huignard, J. P., J. Appl. Phys. 58 (1985) 45.

[39] Stepanov, S. I., Petrov, M., Opt. Commun. 53 (1985) 292.

[40] Peltier, M., Micheron, F., J. Appl. Phys. 48 (1977) 3683.

[41] Herriau, J. P., Rojas, D., Huignard, J. P., BasSAT, J. M., LAUnAY, J. C., soumis à Ferroelectrics.

[42] Kukhtarev, N. V., Markov, V. G., Odulov, S. G., Soskin, M. S., VinetskiI, V. L., Ferroelectrics 22 (1979) 961.

[43] Kogelnik, H., Bell Syst. Tech. J. 48 (1969) 2909.

[44] Glass, A. M., Klein, M. B., Valley, G. C., Elect. Lett. 21 (1985) 220.

[45] Pauliat, G., Allain, M., Launay, J. C., Roosen, G., Opt. Commun. 61 (1987) 321.

[46] Grousson, R., Henry, M., Mallick, S., J. Appl. Phys. 56 (1984) 224.

[47] Strohkendl, F. P., Jonathan, J. M. C., HellWARTH, R. W., Opt. Lett. 11 (1986) 312.

[48] Valley, G. C., J. Appl. Phys. 59 (1986) 3363.

[49] Kukhtarev, N. V., Dougalenko, G. E., Starkov, V. N., Appl. Phys. A. 33 (1984) 227.

[50] Le Saux, G., Roosen, G., Brun, A., Opt. Commun. 56 (1986) 374.

[51] Kostyuk, B. Kh., Yu KudzIn, A., SokolyanskiI, G. Kh., Sov. Phys. Solid State 22 (1980) 1429.

[52] Douglas, G. G., ZitTer, R. N., J. Appl. Phys. 39 (1968) 2133.

[53] Le Saux, G., Launay, J. C., Brun, A., Opt. Commun. 57 (1986) 166.

[54] Le Saux, G., Thèse, Univ. Paris-Sud, 1986.

[55] Ferrier, J. L., Gazengel, J., Nguyen Phu, X., Rivoire, G., Opt. Commun. 58 (1986) 343.

[56] Le Saux, G., Roosen, G., Brun, A., Opt. Commun. 58 (1986) 238.

[57] Huignard, J. P., Marrakchi, A., Opt. Lett. 6 (1981) 22.

[58] Shi, Y., Psaltis, D., Marrakchi, A., Tanguay, A. R., Appl. Opt. 22 (1983) 3665.

[59] Pichon, L., Huignard, J. P., Opt. Commun. 36 (1981) 277.

[60] Ochoa, E., Hesselink, L., Goodman, J., Appl. Opt. 24 (1985) 1826. 
[61] Stepanov, S. I., Petrov, M., Opt. Acta 31 (1984) 1335.

[62] Roosen, G., Plantegenest, M. T., Ferroelectrics 56 (1984) 137.

[63] Rajbenbach, H., Huignard, J. P., Opt. Lett. 10 (1985) 137.

[64] FeInBERg, J., Optical phase conjugation. R.A. Fisher Editor, (Quantum Electronics, Academic Press) 1983, p. 417.

[65] Gaylord, T. K., Mirsalehi, M. M., Guest, C. C., Opt. Eng. 24 (1985) 48.

[66] Psaltis, D., International Optical Computing Conference (Jerusalem) 1986, Proceedings SPIE, Vol. 700 , p. 278.

[67] Goodman, J. W., LeOnberger, FD. I., KeUnG, S. Y., Athale, R. A., Proc. IEEE 72 (1984) 850 .
[68] Pauliat, G., Roosen, G., International Optical Computing Conference (Jerusalem) 1986, Proceedings SPIE, Vol. 700, p. 202.

[69] Pellat Finet, P., Opt. Commun. 50 (1984) 275.

[70] Peltier, M., J. Appl. Phys. 48 (1977) 3683.

[71] Lenzo, P. V., Spencer, E. G., Ballman, A. A., Appl. Opt. 5 (1966) 1688.

[72] Telle, J. M., Tang, C. L., Appl. Phys. Lett. 26 (1975) 572.

[73] Hopfield, J. J., Proc. Nat. Acad. Sci. 79 (1982) 2554.

[74] Mc Cullogh, N. S., PitTs, W., Bull. Math. Biophysiol. 5 (1943) 115.

[75] HeBB, D., Organization of Behaviour (Wiley, N.Y.) 1941.

[76] Strait, J., Glass, A. M., J. Opt. Soc. Am., B 3 (1986) 342. 